\title{
De la Mégère apprivoisée au Roman de Julie Papineau. Origines d'un rituel du mariage franco-ontarien
}

\section{Jean-Pierre Pichette}

Volume 6, 2005

URI : https://id.erudit.org/iderudit/1039333ar

DOI : https://doi.org/10.7202/1039333ar

Aller au sommaire du numéro

\section{Éditeur(s)}

Société Charlevoix

Presses de l’Université d'Ottawa

ISSN

1203-4371 (imprimé)

2371-6878 (numérique)

Découvrir la revue

Citer cet article

Pichette, J.-P. (2005). De la Mégère apprivoisée au Roman de Julie Papineau. Origines d'un rituel du mariage franco-ontarien. Cahiers Charlevoix, 6, 195-248. https://doi.org/10.7202/1039333ar
Résumé de l'article

Jean-Pierre Pichette ajoute un deuxième chapitre à son étude d'une sanction rituelle du mariage franco-ontarien et il se met en quête de l'origine de la « danse sur les bas ». Comme cette tradition est attestée dans les recoins de la francophonie d'Amérique et inconnue des populations anglophones, il se tourne naturellement vers la France. Bien que, là aussi, des expressions métaphoriques et de nombreuses pratiques stigmatisent l'aîné devancé dans le mariage par son cadet, rien de comparable aux rituels canadiens-français. Explorant alors la filière britannique, il débusque, depuis la danse pieds nus de La Mégère apprivoisée de Shakespeare jusqu'à la danse dans l'auge décrite par des folkloristes et des curieux au XIX ${ }^{\mathrm{e}}$ siècle, des parallèles de la plupart des variantes qui ont cours en Amérique française. Cette constatation l'amène à s'interroger sur le cheminement probable de cette coutume, dans laquelle les Bretons, les Celtes français, auraient pu jouer un rôle déterminant de diffuseurs au temps de l'émigration française au Canada. Sans découvrir chez eux les formes qui manifesteraient ce rôle, l'auteur relève néanmoins dans le personnage du baz-valan, ou entremetteur de mariage, de nombreux indices qui appuient son hypothèse : son statut de célibataire, le port de bas colorés et dépareillés dans l'accomplissement de sa charge, et la danse avec la mariée comme récompense. Si l'influence celtique est certaine, son lieu, en France ou au Canada, reste à déterminer.
Ce document est protégé par la loi sur le droit d'auteur. L’utilisation des services d’Érudit (y compris la reproduction) est assujettie à sa politique d'utilisation que vous pouvez consulter en ligne.

https://apropos.erudit.org/fr/usagers/politique-dutilisation/ 


\section{De la MÉGÈre aPPRIVOISÉE Au RoMAN DE Julie Papineau. Origines d'un rituel DU MARIAGE FRANCO-ONTARIEN}

Jean-Pierre Pichette

Département de folklore et ethnologie de l'Amérique française Université de Sudbury, Sudbury et

Chaire de recherche du Canada en oralité des francophonies minoritaires (COFRAM) Centre acadien

Université Sainte-Anne, Pointe-de-l'Église (N.-É.) 


\section{SOMMAIRE}

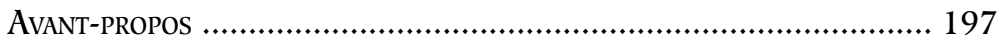

I - UN FOYER QUÉBÉCOIS LACUNAIRE .............................................. 198

A. Les mouvements migratoires ......................................... 198

B. Des dynamismes régionaux ............................................ 203

C. En marge de la culture dominante ................................... 205

II - La SAnction de l’aîné célibataire en France ........................... 207

A. Des expressions ………...................................................... 208

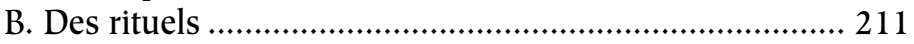

1. Le sachet d'avoine ou les hautons

2. Le tribart

3. La quenouille et l'oignon

4. Le cul du four

5. La cuve

6. La chèvre blanche

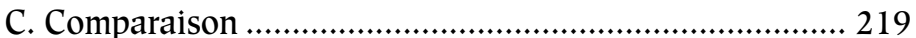

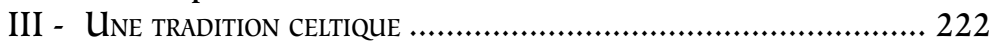

A. La filière irlandaise et britannique ................................. 222

1. La Grande-Bretagne

2. L'Irlande

3. Ailleurs

B. Le baz-valan et la filière bretonne .................................. 238

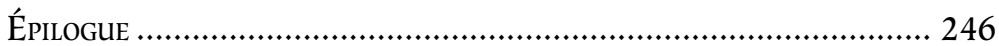




\section{De la MÉGÈre APPRIVOISÉE Au RoMAN DE Julie Papineau. Origines d'un Rituel} DU MARIAGE FRANCO-ONTARIEN

"L'étude d'une aire, sa nature - simple ou composée le dessin de ses contours, sont a priori imprévisibles. L'enquête seule les découvre ${ }^{1}$."

\section{Avant-propos}

Dans la première partie de cette étude ${ }^{2}$, nous avons examiné une sanction populaire très courante dans plusieurs régions de l'Ontario français, dans le Nord particulièrement: «la danse sur les bas » que l'aîné célibataire doit exécuter le jour du mariage de son cadet. À l'aide d'un questionnaire et de nombreuses enquêtes menées en territoire ontarien, nous avons d'abord pu proposer une image assez complète de sa vitalité contemporaine, dater les formes qu'elle a revêtues au cours du dernier siècle danse sur les bas, danse dans une auge ou dans une cuve;

\footnotetext{
${ }^{1}$ Jean-Michel Guilcher, La Tradition populaire de danse en Basse-Bretagne. Nouvelle édition, augmentée d'illustrations et d'un avant-propos, [Chambéry, Imprimeries réunies, 1963], Spézet-Douarnenez, Coop-Breizh-Chasse-Marée/ Armen, 1995, p. X.

${ }^{2}$ Jean-Pierre Pichette, «"Danser sur ses bas". Rémanence d'une sanction populaire dans le rituel du mariage franco-ontarien", Cahiers Charlevoix. Études franco-ontariennes $\mathrm{n}^{\circ} 5$, Sudbury, Société Charlevoix et Prise de parole, 2002, p. 229-311.
} 
port de bas simples ou ornés; jet de pièces de monnaie et cerner son évolution avant de dégager son scénario fondamental et ses caractéristiques générales. Ensuite, par des recherches additionnelles dans les publications et les archives disponibles, et par des sondages en divers milieux de la francophonie nord-américaine, nous avons pu apprécier son ancienneté, attestée à la fin du Régime français et entretenue au fil du temps par la littérature orale, son expansion et ses formes régionales au Québec, en Acadie, dans l'Ouest et aux États-Unis.

Ce premier portrait a ainsi fait apparaître, en plus des variations selon l'espace et le temps, en fréquence ou en durée, en originalité ou en intensité, des zones vierges, où ce rituel du mariage est inconnu, et, singulièrement, au sein de populations qui sont au cour de la francophonie nord-américaine, la capitale du Québec et sa métropole notamment. Ces lacunes, qui ne peuvent être portées au compte d'une enquête sommaire, nous amènent maintenant, dans la deuxième partie de notre étude, à considérer les origines européennes de cette tradition francoontarienne et canadienne-française afin de dépister le réseau des influences possibles et de justifier sa présence ici en suggérant un cheminement probable.

\section{I - UN FOYER QUÉBÉCOIS LACUNAIRE}

\section{A. LES MOUVEMENTS MIGRATOIRES}

Quand on les reporte sur la carte de l'Amérique française, les attestations et les formes de ce rituel livrent quelques renseignements sur leur existence en certains points du continent dont l'analyse pourra tirer profit. Cinq zones se dessinent alors nettement et indiquent des aires de conservation. D'abord, trois zones figurent à l'ouest du Québec: 1. le foyer le plus actif, par sa vitalité, et le mieux représenté par l'effort d'enquête qui y a spécialement été consenti, est indubitablement le nord-est ontarien, avec sa 


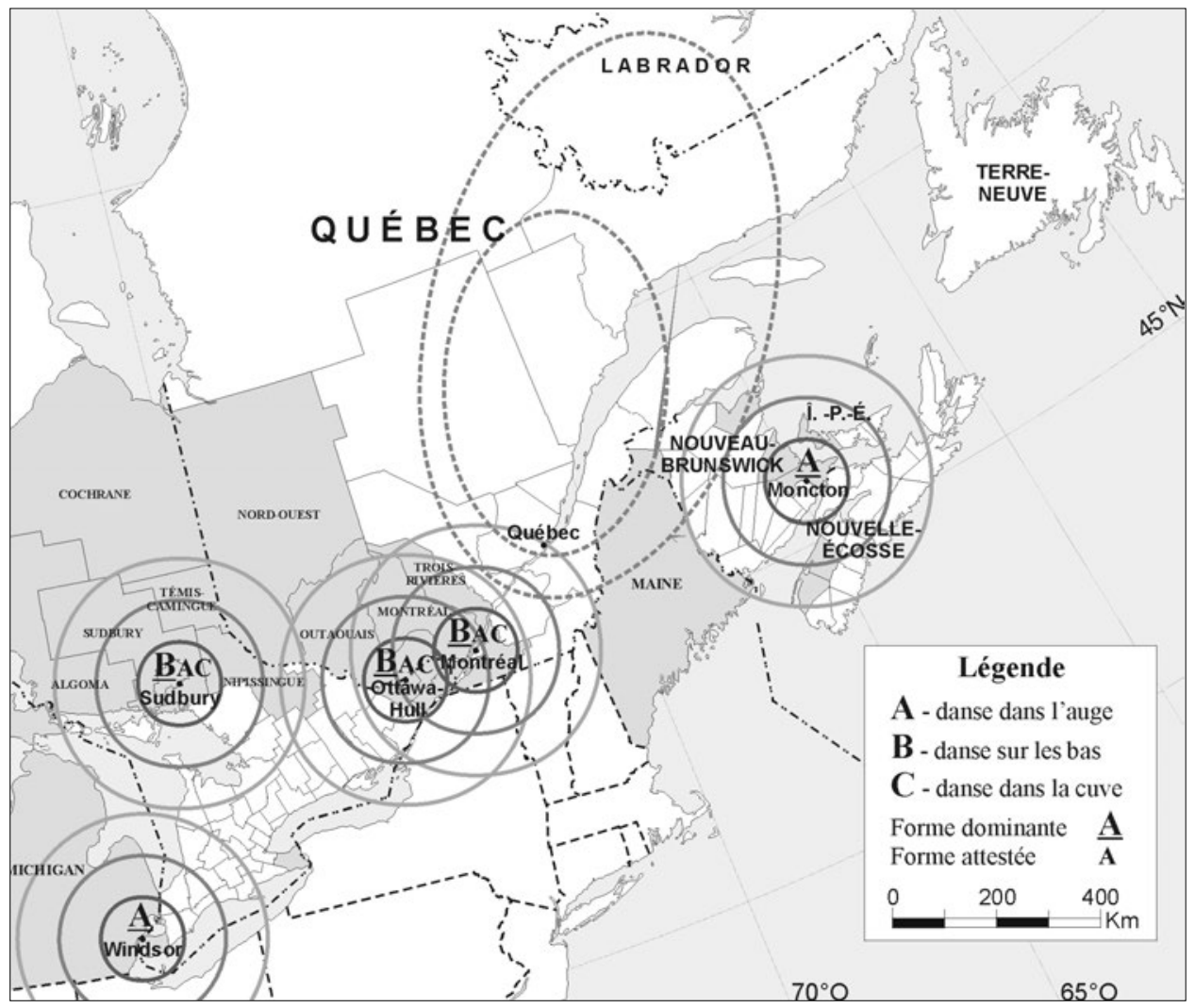

correspondance dans le nord-ouest québécois: y foisonnent toutes les variantes de la danse sur les bas, avec aussi des formes régressives de la danse dans l'auge ou dans une cuve; 2 . l'Outaouais québécois et son débordement ontarien ont retenu la danse sur les bas et dans la cuve; 3 . enfin le sud-ouest ontarien et le Michigan se confinent à la danse dans l'auge. Du côté est, deux zones se profilent plus timidement: 4. l'Acadie des Maritimes, dont le NouveauBrunswick est le maillon le plus résistant, et son prolongement étatsunien, dans le Maine et la Louisiane, se 
restreignent également à l'auge; 5 . en dernier lieu, la vallée laurentienne, dans sa partie ouest, possède des reliquats de la danse sur les bas et de la danse dans l'auge en périphérie de Montréal, mais cette dernière forme ne conserverait sa vigueur que dans le Haut-Richelieu.

\section{TABLEAU 1}

Formes de la danse rituelle de l'aîné célibataire selon les époques et les régions en Amérique française

\begin{tabular}{|c|c|c|c|c|c|}
\hline & 1604 & 1704 & 1804 & 1904 & 2004 \\
\hline \multicolumn{6}{|l|}{ Canada } \\
\hline \multicolumn{6}{|l|}{ OuEst } \\
\hline Manitoba & $\mathrm{X}$ & $\mathrm{X}$ & $\mathrm{X}$ & $\mathrm{X}$ & $\mathrm{X}$ \\
\hline Saskatchewan & $\mathrm{X}$ & $\mathrm{X}$ & $\mathrm{X}$ & $\mathrm{X}$ & {$[\mathrm{A}]$} \\
\hline Alberta & $\mathrm{X}$ & $\mathrm{X}$ & $\mathrm{X}$ & $\mathrm{X}$ & $\mathrm{X}$ \\
\hline Colombie-Britannique & $\mathrm{X}$ & $\mathrm{X}$ & $\mathrm{X}$ & $\mathrm{X}$ & $\mathrm{X}$ \\
\hline \multicolumn{6}{|l|}{ ONTARIO } \\
\hline Nord-Ouest & $\mathrm{X}$ & $\mathrm{X}$ & $\mathrm{X}$ & $\mathrm{X}$ & $\mathrm{X}$ \\
\hline Nord-Est & $\mathrm{X}$ & $\mathrm{X}$ & $\mathrm{X}$ & $\mathrm{X}$ & $\mathrm{ABC}$ \\
\hline Centre & $\mathrm{X}$ & $\mathrm{X}$ & $\mathrm{X}$ & $\mathrm{X}$ & $\mathrm{AB}$ \\
\hline Est & $\mathrm{X}$ & $\mathrm{X}$ & $\mathrm{X}$ & $\mathrm{X}$ & $\mathrm{ABC}$ \\
\hline Sud-Ouest & $\mathrm{X}$ & $\mathrm{X}$ & $\mathrm{X}$ & $\mathrm{X}$ & $\mathrm{AB}$ \\
\hline \multicolumn{6}{|l|}{ QuÉBEC } \\
\hline Nord-Ouest & $\mathrm{X}$ & $\mathrm{X}$ & $\mathrm{X}$ & $\mathrm{X}$ & $\mathrm{AB}$ \\
\hline Outaouais & $\mathrm{X}$ & $\mathrm{X}$ & $\mathrm{X}$ & $\mathrm{X}$ & $\mathrm{ABC}$ \\
\hline Montréal & $\mathrm{X}$ & $\mathrm{X}$ & $\mathrm{B}$ & $\mathrm{X}$ & $\mathrm{ABC}$ \\
\hline Centre & $\mathrm{X}$ & $\mathrm{X}$ & $\mathrm{X}$ & $\mathrm{X}$ & {$[\mathrm{A}][\mathrm{B}]$} \\
\hline Québec & $\mathrm{X}$ & $\mathrm{X}$ & $\mathrm{X}$ & $\mathrm{X}$ & $\mathrm{X}$ \\
\hline Est & $\mathrm{X}$ & $\mathrm{X}$ & $\mathrm{X}$ & $\mathrm{X}$ & $\mathrm{X}$ \\
\hline \multicolumn{6}{|l|}{ ACADIE } \\
\hline Nouveau-Brunswick & $\mathrm{X}$ & $\mathrm{X}$ & $\mathrm{X}$ & $\mathrm{X}$ & A \\
\hline Nouvelle-Écosse & $\mathrm{X}$ & $\mathrm{X}$ & $\mathrm{X}$ & $\mathrm{X}$ & A \\
\hline Île-du-Prince-Édouard & $\mathrm{X}$ & $\mathrm{X}$ & $\mathrm{X}$ & $\mathrm{X}$ & {$[\mathrm{A}]$} \\
\hline Terre-Neuve & $\mathrm{X}$ & $\mathrm{X}$ & $\mathrm{X}$ & $\mathrm{X}$ & $\mathrm{X}$ \\
\hline \multicolumn{6}{|l|}{ États-Unis } \\
\hline Michigan & $\mathrm{X}$ & $\mathrm{X}$ & $\mathrm{X}$ & $\mathrm{X}$ & A \\
\hline Maine & $\mathrm{X}$ & $\mathrm{X}$ & $\mathrm{X}$ & $\mathrm{X}$ & A \\
\hline Louisiane & $\mathrm{X}$ & $\mathrm{X}$ & $\mathrm{X}$ & $\mathrm{X}$ & {$[\mathrm{A}]$} \\
\hline
\end{tabular}

LÉGENDE: A: danse dans l'auge; B: danse sur les bas; C: danse dans la cuve; $\mathrm{X}$ : forme inconnue; et $[\mathrm{A}]$ ou $[\mathrm{B}]$ : une expression rappelle cette forme du rituel 
La répartition de ce rituel en ces divers pays s'explique assez naturellement par certains courants de colonisation et de migration qui ont eu lieu à l'intérieur du continent au cours des siècles; ces mouvements de la population française ont emprunté deux axes principaux, mais non exclusifs, l'un, d'est en ouest, et l'autre, du nord au sud: a) au temps de la Nouvelle-France, aux XVII ${ }^{e}$ et XVIII ${ }^{e}$ siècles, une impulsion initiale vers l'ouest, à partir de Québec, a ouvert la colonisation du continent jusqu'au Détroit et, de là, a gagné la Louisiane, en direction sud; b) une marche d'est en ouest aux XIX ${ }^{e}$ et $\mathrm{XX}^{\mathrm{e}}$ siècles a peuplé l'Outaouais, l'est et le nord-est de l'Ontario, puis, en direction nord, a joint l'Abitibi et le Témiscamingue; c) chez les Acadiens, le "Grand Dérangement» a provoqué un exode forcé vers le sud au XVIII ${ }^{e}$ siècle, suivi d'un retour fragmentaire vers le nord, puis, aux XIX ${ }^{e}$ et $\mathrm{XX}^{\mathrm{e}}$ siècles, d'un déplacement vers l'ouest.

L'importante saignée migratoire des Canadiens français vers les États-Unis, au milieu du XIX ${ }^{\mathrm{e}}$ siècle, semble n'avoir laissé que bien peu de traces de cette tradition dans les nouveaux établissements; à la rigueur, les attestations du Maine et du Michigan pourraient lui être attribuées, mais ces dernières s'expliqueraient tout aussi bien par des influences acadiennes et franco-ontariennes ou des reliquats du Régime français. À la même époque, les migrations intérieures vers le Saguenay-Lac-Saint-Jean, comme les mouvements de colonisation de la Gaspésie et des Cantons de l'Est, auraient produit des résultats similaires. Cette déficience peut se comprendre par la zone centrale demeurée vierge sur la carte: l'est de la vallée laurentienne, comprenant les peuplements de Québec et de Trois-Rivières, avec ses prolongements dans le nord-est du Québec (SaguenayLac-Saint-Jean, Côte-Nord) comme aussi sur la côte sud et en Gaspésie, se démarque donc par son mutisme. $\mathrm{Si}$, au XIX $^{e}$ siècle, cette danse traditionnelle n'existait pas, ou n'était plus en usage, dans cette zone fondatrice de départ, 
on n'avait bien évidemment pas pu la transmettre au terme de la migration.

Ainsi, la plus ancienne aire de peuplement français d'importance en Amérique, la ville de Québec et sa région, n'en a gardé absolument aucun souvenir, comme d'ailleurs la majeure partie de l'est québécois. La deuxième, la région de Trois-Rivières, n'en a guère conservé que de vagues rappels en périphérie. Et la troisième, la région de Montréal, qui n'en a rien préservé dans sa partie urbaine, présente quelques attestations dans les banlieues qui la ceinturent. Comme il appert par la lettre écrite en 1826 par Joseph Papineau et que nous avons analysée au préalable ${ }^{3}$, on peut sans danger supposer que cette tradition était déjà connue dans l'île de Montréal à la fin du XVIII ${ }^{\mathrm{e}}$ siècle. Non attestée pendant plus d'un siècle, elle ne subsisterait plus qu'en périphérie où elle s'est maintenue jusqu'à nos jours ${ }^{4}$. En nette régression dans la première moitié du $\mathrm{XX}^{\mathrm{e}}$ siècle ${ }^{5}$, cette tradition aurait suivi au Canada une trajectoire comparable à celle qu'un certain nombre de traditions françaises ont suivie chez nous après avoir été oubliées en France, leur pays d'origine ${ }^{6}$. Elle survivrait dans les marches françaises, en marge plutôt qu'au centre, comme l'écho périphérique contemporain d'une impulsion centrale ancienne.

Car, si l'Acadie, la Louisiane, le Maine, le Québec, l'Ontario et le Michigan connaissent tous la danse dans l'auge, d'une part, c'est que cette forme aurait dû irradier à partir d'un foyer commun dès le XVII ${ }^{\mathrm{e}}$ ou le XVIII ${ }^{\mathrm{e}}$ siècle, à l'époque où la Nouvelle-France liait, sur les plans politique, économique et culturel, ces collectivités dispersées

${ }^{3}$ Ibid., p. 246-250.

${ }^{4}$ Ibid., p. 285-287.

${ }^{5}$ Ibid., p. 299.

${ }^{6}$ Dans notre article «La Diffusion du patrimoine oral des Français d'Amérique» (dans Langue, espace, société. Les Variétés du français en Amérique du Nord, sous la direction de Claude Poirier et al., Sainte-Foy, Les Presses de l'Université Laval, coll. «Culture française d'Amérique», 1994, p. [127]-143), nous rapportons des exemples pour le conte, la chanson et les jurons. 
sur le continent nord-américain ${ }^{7}$. D'autre part, le fait que le Québec et l'Ontario connaissent seuls la danse sur les bas témoignerait que cette tradition est une variation régionale, plus tardive peut-être, du rituel. Mais, attestée une première fois en 1826 et remontant au XVIII ${ }^{\mathrm{e}}$ siècle, elle pourrait être aussi ancienne que la danse dans l'auge. Toutefois, les mouvements vers l'ouest, principalement à partir de la région de Montréal, auraient emporté en Abitibi, dans l'Outaouais, mais combien plus dans le nord-est ontarien, les germes du rituel contemporain. Anémiée au centre, sinon éteinte au siècle suivant, on ne la relèverait plus maintenant que dans les marges plus rurales autour.

\section{B. DES DYNAMISMES RÉGIONAUX}

Néanmoins, l'indice limité de ce rituel au Québec, résultat de son étiolement présumé, conduit inévitablement à s'interroger sur une possible influence acadienne dans le Nord ontarien, hypothèse que n'ont jamais formulée les études régionales de l'Acadie ou de l'Ontario. Ainsi une région périphérique aurait-elle pu influencer une autre région de cette même périphérie sans passer par le centre? L'Acadie auraitelle pu influencer directement l'Ontario français?

On a déjà remarqué que Sudbury est depuis le début du $\mathrm{XX}^{\mathrm{e}}$ siècle une capitale régionale, située à un carrefour, vers laquelle ont convergé des familles de toutes les régions françaises du Canada, et même du nord des ÉtatsUnis. Les Acadiens y sont ainsi très bien représentés, comme en fait foi le grand nombre de familles portant leurs si caractéristiques patronymes - Arsenault, Benoît, Blanchard, Boudreau, Cormier, Ferron, Landry, Leblanc, Maillet et Richard, pour ne citer que les plus courants dans cette région.

\footnotetext{
${ }^{7}$ Des questions demeurent pourtant, comme cette remarque d'Yves Frenette nous le suggérait dans une note privée du printemps 2002: «Il est significatif qu'on ne trouve pas de trace de ce rituel à la Rivière-aux-Canards, une localité de peuplement plus ancien, un peuplement canayen plutôt que canadien-français ».
} 
Même si l'installation de familles acadiennes rend virtuellement possible une telle influence, cette hypothèse paraît bien improbable, en ce qui concerne l'exemple à l'étude tout au moins, étant donné que les familles francoontariennes d'origine québécoise connaissent cette tradition souvent mieux que les familles d'origine acadienne, qu'on l'a aussi relevée très vivante dans la région de Hearst où il n'est à peu près pas venu d'Acadiens et qu'on a trouvé des traces de ce rituel dans la région de l'Outaouais et de Montréal d'où sont issues bon nombre des familles francoontariennes. De plus, la "danse dans l'auge», variante de ce rituel et seule forme attestée un peu partout dans les divers pays de l'Acadie, est marginale dans le nord de l'Ontario et vraisemblablement en déclin. L'idée d'une influence acadienne directe ne serait pas réaliste.

Néanmoins, depuis la période du "Grand Dérangement » au XVIII ${ }^{e}$ siècle, de nombreuses familles acadiennes déportées ont trouvé refuge un peu partout au Canada et aux États-Unis, et ont parfois reconstitué de véritables communautés acadiennes. Les ramifications de cette diaspora sont aujourd'hui bien connues: en plus des Cadiens qui se sont rassemblés en Louisiane et de ceux qui se sont réinstallés sur les côtes de toutes les provinces atlantiques (NouveauBrunswick, Nouvelle-Écosse, Île-du-Prince-Édouard et aussi Terre-Neuve), d'autres se réfugièrent au Québec (notamment aux Îles-de-la-Madeleine, en Gaspésie, sur la côte nord du Québec et dans la région de Montréal) ou dans le Maine ${ }^{8}$. La présence de ces Acadiens aurait-elle contribué à la diffusion ou au renforcement de la forme unique du rituel que l'Acadie connaît, la danse dans l'auge? L'installation d'une population acadienne dans la vallée du Richelieu, là où précisément se concentre aujourd'hui encore, avec une réelle vitalité, cette forme particulière, aurait-elle pu produire un impact aussi grand? Le village

${ }^{8}$ Maurice Basque et al., L'Acadie de l'Atlantique, Moncton, Centre d'études acadiennes (SNA, CÉA, CIDEF-AFI), 1999, p. 39. 
de L'Acadie, dont le nom si manifeste rappelle qu'un contingent de plus de 500 Acadiens s'y est établi à la fin du XVIII ${ }^{e}$ siècle, pourrait en être l'indice, mais il est impossible de le mesurer.

En somme, si ces régions périphériques ne semblent pas s'être mutuellement influencées, mais bien l'avoir été par les régions de l'ouest du Québec pour l'Ontario, il serait donc possible que le centre ait délaissé cette tradition qui, conservant tout son dynamisme et sa pertinence en périphérie, s'y serait renouvelée.

\section{EN MARGE DE La Culture dominante}

Le statut minoritaire des Acadiens et des FrancoOntariens, qui se traduit par une grande perméabilité à la culture dominante, oblige en outre à considérer les traditions anglophones d'Amérique avant de sonder les sources européennes de chacun de ces terroirs. Ces deux populations minoritaires, l'acadienne et la franco-ontarienne, auraient-elles subi l'influence des majorités anglo-saxonnes qui les dominent? Cette hypothèse justifierait à la fois la rareté de ce rituel dans les régions centrales du Québec (Québec, Trois-Rivières et Montréal), là où la population française est majoritaire, et sa présence dans les zones minoritaires de la périphérie, là où elle est en relation constante avec la majorité anglophone.

Or, vérification faite, une telle infiltration de la culture dominante dans le rituel du mariage des minorités françaises n'aurait jamais eu lieu. De nombreuses consultations privées - une cinquantaine peut-être -, menées tout au long de cette étude, ont constamment abouti au même résultat: ce rituel du mariage est généralement inconnu des anglophones nord-américains. En 1999 et 2000, six de nos étudiants ${ }^{9}$ ont $\mathrm{pu}$, dans le cadre de leurs travaux

${ }^{9}$ Estelle Corriveau, Fritz-Gérald Destiné, Annie Gagné, Sophie Laflamme, Eding Mvilongo et Jacinthe Trudeau. 
de terrain, valider ces observations auprès d'une quinzaine de répondants anglophones, vivant dans trois provinces canadiennes (Terre-Neuve, Québec, Ontario), et de quelques hispanophones ${ }^{10}$. Des chercheurs, ethnologues et historiens bien au fait des traditions de Terre-Neuve, de la Nouvelle-Écosse, de l'Ontario, du Manitoba et de la Colombie-Britannique, dont nous avons sollicité les avis, ont aussi tous corroboré ces données ${ }^{11}$. L'un d'entre eux est Peter Ward, l'auteur d'une étude sur le mariage chez les Anglo-Canadiens au XIX ${ }^{e}$ siècle $^{12}$. Les remarques que ce spécialiste nous communiqua valent qu'on les rapporte ici, en raison du dépouillement général qu'il dut accomplir pour sa propre recherche et, partant, de l'autorité qu'elles revêtent pour la nôtre.

Votre projet me semble fascinant et j'en suis curieux. [...] Sauf que, durant la recherche que je fis pour ce projet, pas une seule fois n'ai-je rencontré une description de la «danse sur les bas». Puisque c'était tout à fait le type d'usage que je recherchais, je puis affirmer avec certitude qu'elle n'a jamais été une coutume d'importance au Canada anglais. Je ne peux éclairer son origine ${ }^{13}$.

Cette attestation négative confirmait les avis de collègues ethnologues anglophones et les sondages menés auprès de la population anglophone du nord de l'Ontario et d'ailleurs. En somme, les seuls anglophones qui connaissent cette tradition vivent dans le voisinage des Franco-Ontariens ou ont assisté à des noces canadiennes-françaises. De plus, les

\footnotetext{
${ }^{10}$ Ce groupe comprenait un nombre à peu près égal d'hommes (9) et de femmes (8), nés entre 1923 et 1980 - soit 8 adultes nés entre 1923 et 1974 et 9 étudiants nés entre 1976 et 1980 - , anglophones du Canada (15) et hispanophones du Mexique et du Guatemala (2).

${ }^{11}$ Il s'agit de Pauline Greenhill et de Carol Harvey, de l'Université de Winnipeg, de Diane Tye, de l'Université Memorial, et de Peter Ward, de l'Université de la Colombie-Britannique.

${ }^{12}$ Peter Ward, Courtship, Love and Marriage in Nineteenth-Century English Canada, Montréal \& Kingston, London, Buffalo, McGill-Queen's University Press, [1990], 219 p.

${ }^{13}$ Traduction de l'auteur du courriel de Peter Ward, département d'Histoire, Université de la Colombie-Britannique, Vancouver, le 3 août 1999.
} 
Franco-Ontariens valident aussi ce fait, percevant clairement qu'il s'agit bel et bien d'une coutume canadiennefrançaise. C'est également l'opinion de Peter Ward qui avance qu'elle «pourrait aisément s'être développée à l'intérieur de la société canadienne-française elle-même». Celui-ci précise encore que, dans le petit nombre «d'études consacrées au mariage et à l'ordre de naissance dans les familles de l'époque coloniale en Amérique», on ne «fait aucune mention de la coutume qui vous intéresse ${ }^{14} »$. Cette constatation rejoint celle de Michael Taft qui fit compulser, à notre demande, les archives de la culture populaire de l'American Folklife Center à Washington: «Je n'ai jamais entendu parler de cette tradition ni de rien de semblable ${ }^{15}$."

Dès lors, il s'agirait bien d'une sanction populaire propre aux communautés d'origine canadienne-française d'Amérique. Attestée de nos jours dans presque tous les recoins de sa diaspora, elle n'a pu se répandre qu'à partir d'une source canadienne commune, probablement dès les premiers temps de la Nouvelle-France. Son absence de nos jours dans les grands centres du Québec serait le résultat d'une érosion déjà ancienne, particulièrement dans le Centre et l'Est, antérieure en tout cas aux migrations du XIX siècle; tandis que l'épanouissement spectaculaire que nous avons observé dans la partie ouest du Québec, notamment dans le nord-est ontarien, serait la résurgence d'une tradition montréalaise en déclin.

\section{II - La sanction de l'aîné célibataire en France}

Si tant est que cette sanction populaire remonte aux premiers habitants de la Nouvelle-France, la source pourrait peut-être se retrouver du côté de la mère patrie. Cette danse rituelle a-t-elle été consignée en France autrefois?

\footnotetext{
${ }^{14}$ Loc. cit.

${ }^{15}$ Michael Taft, chef, Archive of Folk Culture, American Folklife Center, Bibliothèque du Congrès, Washington, courriel du 28 janvier 2004.
} 
Sinon, la sanction de l'aîné célibataire y a-t-elle déjà existé et sous quelle forme? Les grands travaux d'Arnold Van Gennep aideront, avec ceux d'autres folkloristes européens, à répondre à cette question.

\section{A. DES EXPRESSIONS}

En France comme au Canada, l'usage prescrit qu'il faut d'abord marier l'aîné puis le cadet. Procéder autrement passe alors pour une anomalie que stigmatisent de nombreuses expressions populaires. Arnold Van Gennep, dans la section de son Manuel de folklore français contemporain qu'il a consacrée au "mariage du cadet ou de la cadette $^{16}$ ", relève plusieurs de ces formules métaphoriques qui marquent le renversement de la normalité. Ainsi, marier la cadette avant l'aînée, c'est « tourner la queue du pot vers les tisons" en Gascogne; c'est "décuveler la lessive", "c'est-à-dire laver d'abord le linge du dessous du cuvier» en pays messin; c'est "faucher l'avoine avant les blés" à Iwuy (Nord) ${ }^{17}$; c'est "écoupeler (étêter) les chou $^{18}$ " ou "couper l'herbe sous le pied de l'aîné» en Bretagne; c'est "manger le chou " à Grissey et à Neuil (Indre-et-Loire $)^{19}$; c'est encore « manger une tarte retournée», ou la lui offrir, ou tout au moins un morceau... (la Somme, Saint-Pol en Ternoise, Pas-de-Calais, toute la région de Lille) ${ }^{20}$. De nos jours, au tournant $\mathrm{du} \mathrm{XXI}^{\mathrm{e}}$

\footnotetext{
${ }^{16}$ Arnold Van Gennep, Manuel de folklore français contemporain, Paris, Éditions A. et J. Picard, tome I, vol. 2, Du berceau à la tombe (fin). MariageFunérailles, 1946, p. 628-635.

${ }^{17}$ Ibid., tome I, vol. 2, p. 629.

${ }^{18}$ Voir aussi "étêter les choux» (1901) dans Revue de folklore français, Paris, Librairie Larose, tome V, $\mathrm{n}^{\text {os }} 4-5$, juillet-octobre 1934, p. 305-306, «Le Mariage de la cadette", d'après l'Intermédiaire des chercheurs et des curieux, vol. XLIII (1901), p. 442.

${ }^{19}$ Van Gennep, op. cit., tome I, vol. 2, p. 629: «"la cadette a mangé le chou"; aussi présente-t-on au cours du repas à l'aîné ou à l'aînée une tige de chou enrubannée».

${ }^{20}$ Ibid., tome I, vol. 2, p. 630.
} 
siècle, on signale la persistance de ces expressions et d'autres du même genre: en Bretagne, marier d'abord la cadette se disait encore "écoupler les chou ${ }^{21}$ » ou « commencer le tas de paille par le milieu ${ }^{22} »$; en Beauce, c'est "déf[aî]ter le chaumier ${ }^{23}$ ".

D'autres locutions interpellent plutôt l'aînée supplantée. Certaines évoquent son inutilité, sa mise aux rebuts ou sa stérilité. Selon Van Gennep, on dit à Menton que la cadette "a laissé ses sœurs aînées sur la cendre»; dans le Maine qu'elle "fait filer à l'aînée des copeaux" ${ }^{24}$; en Champagne, dans la région de Troyes, qu'elle «l'a mis[e] sous la tinette (petite cuve)»; en Faucigny (Haute-Savoie), que le cadet ou la cadette "a mis l'aîné ou l'aînée sous le van " ${ }^{25}$. Vers 1850, dans le Perche, on rapporte que la cadette « $a$ enruché la vieille», c'est-à-dire: "On fait allusion à une personne à qui on mettrait une ruche sur la tête pour lui empêcher de voir les amans ${ }^{26} »$. La bêtise de l'aînée est aussi évoquée par la formule "faire manger de l'avoine »expression fort répandue au Canada et attestée depuis 1810 au sens de supplanter son rival ${ }^{27}$ - qui la rabaisse au

${ }^{21}$ Daniel Giraudon, courriel du 15 septembre 2000, témoignage d'une femme de Plenéne Jugon, 90 ans.

${ }^{22}$ Daniel Giraudon, courriel du 25 février 2002: «En ti-mãn, ne vez ket boulc'het ar bern kolo dre e greiz » [Dans cette ferme, on ne commence pas le tas de paille par le milieu].

${ }^{23}$ Marie-Rose Simoni-Aurembou, courriel du 21 septembre 2003: d'après un agriculteur de Tivernon (ouest du Loiret, tout près de la limite de l'Eure-etLoir), nommé Serge Dufour, auteur de livres de souvenirs, entre autres «En Beauce. Le vieux parler», s.l., éd. A.A., 1987. Voici ce qu'il dit: «Fallait jamais "dèfâiter l'chaumier". S'il y avait dans la maison plusieurs filles à marier, il fallait que ce soit l'aînée qui "parte la première" "(p. 116). "Défaîter l'chaumier: enlever la couverture de la meule (les meules étaient couvertes en paille, comme les maisons)".

${ }^{24}$ Van Gennep, op. cit., tome I, vol. 2, p. 629.

${ }^{25}$ Loc. cit.

${ }^{26}$ Marie-Rose Simoni-Aurembou, «L'Alphabet du quotidien. Petite chronique du canton de Thiron au XIX ${ }^{\mathrm{e}}$ siècle», par Arsène Vincent (1831-1881), dans les Cahiers percherons $\mathrm{n}^{\circ}$ 69-70, Association des amis du Perche, 1982, p. 51. ${ }^{27}$ Jean-Pierre Pichette, " "Danser sur ses bas". Rémanence d'une sanction populaire dans le rituel du mariage franco-ontarien», op. cit., p. 303-308. 
rang des bêtes: en Ariège, on dit que « la cadette fait manger de l'avoine à l'aînée». Nos correspondants nous en ont rapporté des équivalents contemporains pour la Bretagne: à Mael-Carhaix, on disait à la cadette: "Tu lui as donné un sac d'avoine ${ }^{28} "$; à Baud, dans le Morbihan, que le cadet "donnait à son aîné une fourchée d'épines ${ }^{29} »$; et, dans le Bas-Léon, que l'aînée "porte le fagot d'ajoncs ", c'est-à-dire la nourriture des chevaux ${ }^{30}$. Il est déjà évident que plusieurs de ces expressions sont à mettre en parallèle avec les marques de refus destinées à éconduire un prétendant indésirable ou l'entremetteur qui le représente et dont plusieurs persistent de nos jours comme en témoignent les documents suivants. En 1963, dans son ouvrage capital sur La Tradition populaire de danse en Basse-Bretagne, JeanMichel Guilcher notait, à propos du savoir-vivre, qu'une jeune fille qui refusait de danser avec un garçon lui faisait affront et qu'on disait de celui-ci qu'il avait « reçu son sac », "un sac de blé noir», "plein son chapeau d'avoine», «un taureau tacheté $»^{31}$. Il observait le même comportement au cours d'une noce: « chacun se garde de danser avec d'autres que son partenaire attitré. Ce serait lui donner "un sac d'avoine" (Arradon), c'est-à-dire lui faire injure ${ }^{32} »$. Le pain et l'avoine, entre autres pratiques, étaient aussi présents dans les rites de refus en Wallonie ${ }^{33}$.

${ }^{28}$ Daniel Giraudon, courriel du 19 octobre 1999: «roet peus ur sac'h kerc'h dezhi».

${ }^{29}$ Daniel Giraudon, courriel du 23 novembre 1999: «Si le cadet se mariait avant l'aîné, on disait de l'aîné: ur fourchaf drein zo bet roet dehon [On lui a donné une fourchée d'épines]. Ce n'était valable que pour les garçons. Pour les filles, on était content de s'en débarrasser».

${ }^{30}$ Dicton rapporté par Jean-François Simon et noté à Brest le 4 mars 2002.

${ }^{31}$ Jean-Michel Guilcher, op. cit., p. 44.

32 Ibid., p. 48.

${ }^{33}$ Eugène Monseur, "Coutumes. I. - Les Noces", dans Bulletin du folklore wallon, Liège et Bruxelles, vol. III, tome 2, $1^{\mathrm{er}}$ fascicule, janvier-mars 1893, p. 33 : «38. - Le jeune homme refusé [...]. B. On dit à Liège, non seulement à un jeune homme dédaigné, mais aussi à une jeune fille dont le "galant" se marie avec une autre: n'aléf nin r'kwèri vo mitch? "n'allez-vous pas rechercher vos miches?" On dit au jeune homme à Laroche: vo-z îré ki vos mitch "vous irez 
«Monter sur le cul du four » pourrait avoir le même sens puisque c'est vraisemblablement derrière le four qu'on rangeait certains objets inutiles qu'on voulait mettre au rancart $^{34}$. Selon Van Gennep:

Dans la Somme, vallées de l'Yères et de la Bresle et plateaux intermédiaires, dans les régions de Fruges et de Saint-Omer (Pas-de-Calais), dans celles de La Bassée (Nord), le Hainaut, le Brabant wallon, l'Ardenne et le Luxembourg belges, il ne reste plus qu'une expression qui varie légèrement selon les localités. On dit: que l'aînée doit «danser sur le cul du four», ou doit être "portée sur la voûte», ou la "culotte du four »; cette expression est connue dans presque tout le Pas-de-Calais et le Nord, sans qu'on puisse de nos jours en donner une explication ${ }^{35}$.

\section{B. DES RITUELS}

\section{Le sachet d'avoine ou les hautons}

Jusqu'ici, les exemples se sont limités à des expressions métaphoriques, comme il s'en est aussi rencontré en Amérique française lorsque le rituel avait cessé de se perpétuer et que de telles formules en prolongeaient la mémoire. Mais on a également signalé des rituels qui visaient à ne pas laisser passer inaperçue pareille transgression. Van Gennep note qu'en Isère on présentait à l'aîné " pendant le repas de noces une bouillie d'avoine ${ }^{36}$ ". La même idée de moquerie subsiste dans le port par l'aîné d'un sachet d'avoine au cou ou à la taille, ou d'un sac de paille sur l'épaule. Eugène Monseur parle alors de «porter les

rechercher votre miche". (Refonte de FW 717). [...] D. À Herve, on envoie un petit pain couvert de moutarde ou entouré d'un crêpe noir au jeune homme dédaigné par une jeune fille, le jour du mariage de celle-ci (FW 716)».

${ }^{34}$ Van Gennep, op. cit., tome I, vol. 2, p. 633. Rejetant l'opinion de P. Saintyves, qui y lit un geste de nature érotique, l'auteur se range à l'avis de $\mathrm{M}^{\text {lle }}$ Leroy et interprète cette expression comme une mise au rebut de l'aîné puisque «c'est sur le dessus du four que dans ces régions on jette ou place les objets, vêtements, etc., de rebut».

${ }^{35}$ Ibid., p. 632.

${ }^{36}$ Ibid., p. 630. 
hautons», un rituel qu'il décrit ainsi d'après une note de Jules Guillain:

Dans le sud du Luxembourg, si le marié ou la mariée ont des frères ou sœurs plus âgés et encore célibataires, ces derniers "portent les hautons», c'est-à-dire que pendant le repas de noces, on leur attache en cachette, à la blouse ou à la taille, un petit sac contenant de la balle d'avoine ou des «hautons" (épis battus). La farce est complète si on peut les amener à danser avec leur sac sans qu'ils s'en aperçoivent. Ils sont alors tenus de payer à boire à toute la société et on leur adresse des plaisanteries salées $^{37}$.

Deux ans plus tard, en 1895, dans un nouvel article écrit en collaboration avec Alfred Haron, le même auteur cite une autre attestation: "Se marier avant ses frères ou sœurs aînés, c'est leur "faire porter la hotte", dit-on à Hautfays (province de Luxembourg, arrondissement de Neufchâteau) ${ }^{38}$ ». Selon Roger Pinon, "les hautons symbolisent la stérilité des aînés célibataires». À son tour, Van Gennep mentionne ce rituel ajoutant que, dans «la région de Charleville, on attache un sachet de son au cou de l'aîné », tandis "qu'à Breux, dans la Meuse, l'aîné ou l'aînée doit porter un sac de quarante à cinquante litres de menue paille qu'on lui met sur l'épaule à la fin du dîner ou au milieu du bal ${ }^{39} »$. Cette pratique était toujours à l'honneur en Bretagne il y a quelques décennies ainsi qu'on nous l'a rapporté: "Hier, un ami m'a dit qu'en 1970, à Lescouet sur Meu, on avait passé une ceinture de saule

\footnotetext{
${ }^{37}$ Eugène Monseur, «Coutumes. I. - Les Noces», dans Bulletin du folklore wallon, Liège et Bruxelles, vol. III, tome 2, $1^{\text {er }}$ fascicule, janvier-mars 1893, p. 25 "Comm. de M. Jules Guillain précisant sa brochure Mours luxembourgeoises $18 »$. Texte repris dans la Revue de folklore français, Paris, Librairie Larose, tome V, $n^{\text {os }} 4-5$, juillet-octobre 1934, p. 305-306: "Le Mariage de la cadette" avec cette remarque: «Dans ce dernier cas, le frère aîné ou la sœur aînée sont assimilés symboliquement à des épis vides de grains ou à la balle d'avoine, afin de souligner leur infécondité».

${ }^{38}$ Alfred Haron et Eugène Monseur, dans le Bulletin de folklore, II, 1895, 6, p. 273, no 61. Référence fournie par Roger Pinon.

${ }^{39}$ Van Gennep, op. cit., tome I, vol. 2, p. 630.
} 
autour de la taille de l'aîné le soir des noces de son frère $\operatorname{cadet}^{40} »$.

\section{Le tribart}

On peut rapprocher de cette moquerie la coutume de présenter à l'aîné le tribart, c'est-à-dire l'enfarge ou le collier de bois qu'on passe au cou des bêtes pour les maintenir dans leur clos. Voici ce qu'a recueilli Jacqueline Hautebert dans la région de Nantes, en Loire-Atlantique:

Au cours du repas (Ancenis, Couffé, Anetz, etc...), une curieuse coutume prend parfois place. Si un frère ou une sœur plus âgés [sic] n'est pas encore marié, il doit se présenter avec le tribart. C'est un triangle formé de trois barres de bois, que l'on met au cou des chèvres, des moutons pour les empêcher d'entrer dans les champs. Or, ce jour, c'est la fille non mariée qui arrive, au cours du repas, portant le tribart, enrubanné et garni de fleurs. Signe d'infamie qu'il faut supporter avec le sourire. Des bonbons sont accrochés au tribart et les enfants doivent venir les prendre. Le tribart est parfois remplacé par une quenouille que le ou la délaissée doit faire le simulacre de filer ${ }^{41}$.

\section{La quenouille et l'oignon}

Dans la région de Rennes, en Anjou, en Touraine et en Vendée, Van Gennep rapproche «de l'expression "monter en quenouille" ou "en graine", au sens de prendre de l'âge sans avoir réussi à se marier », les variantes d'un rituel qui implique une quenouille que l'aîné doit filer puis qu'on enflamme $^{42}$. Selon Paul Fortier-Beaulieu, c'est à l'oignon qu'on avait recours dans le département de la Loire pour tirer des regrets aux aînés et les faire pleurer. "Dans le Haut-Forez, une salade d'oignons, d'orties ou même de papier est servie aux frères et sœurs des mariés restés

${ }^{40}$ Daniel Giraudon, courriel du 19 octobre 1999.

${ }^{41}$ Jacqueline Hautebert, "Superstitions de mariage en pays Nantais (LoireAtlantique, Bretagne)», dans Société de mythologie française, Mélanges de mythologie française offerts au président-fondateur Henri Dontenville, Paris, G.-P. Maisonneuve et Larose, 1980, p. 154-155. Référence communiquée par Francine Brunel-Reeves, courriel du 25 octobre 2002.

${ }^{42}$ Van Gennep, op. cit., tome I, vol. 2, p. 631. 
célibataires pour leur faire honte et les faire pleurer, tout en glorifiant les mariés ${ }^{43}$ ». Les ingrédients de la salade présentée à l'aîné, pendant le repas de la noce, peuvent varier: "salade composée d'oignons crus, de chardons, d'orties, de dents de lion", ou une salade de racines appelée «la Chevisse» ou "la Chavissa». On disait alors du cadet: "Il lui a fait manger la salade» ou "Il lui a fait manger la rave». Selon l'auteur, la coutume proviendrait du Vivarais ${ }^{44}$. Dans le Languedoc, tantôt on contraint l'aîné à manger un gros oignon, sans salade cependant, tantôt on " envoie un oignon enrubanné à la plus âgée des sœurs de la mariée ou à une de ses amies si elle est la plus jeune et la première à se marier ${ }^{45}$ "; parfois, oubliant la nature de cette tradition, la mariée elle-même ou ses frères et sœurs plus jeunes mangeaient l'oignon. Il y avait apparemment une raison à ce manège, selon le témoin Albert Hugues:

Dans le Gard, ce n'est pas seulement la sœur aînée qui doit manger de l'oignon au repas de noce de sa cadette, mais le ou les frères aînés. Un énorme oignon leur est servi; ils doivent en déguster une partie, ce qui permet aux jeunes filles assistant au mariage de les refuser pour cavaliers quand elles sont invitées à danser: "Escarta-vous, sentès à séba!" (Écartez-vous, vous sentez l'oignon!) ${ }^{46}$.

4. Le cul du four

$\mathrm{Si}$, comme nous l'avons vu plus haut, il ne restait, dans la première moitié du $\mathrm{XX}^{\mathrm{e}}$ siècle, qu'une expression asso-

${ }^{43}$ Paul Fortier-Beaulieu, Mariages et noces campagnardes dans les pays ayant formé le département de la Loire, Roannais - Forez - partie du Beaujolais Jarez, Paris, Librairie orientale et américaine, Gustave-Paul Maisonneuve, éditeur, 1937, p. 296.

${ }^{44}$ Ibid., p. 297. Van Gennep mentionne aussi cette tradition: op. cit., tome I, vol. 2, p. 632.

${ }^{45}$ Claude Seignolle, Le Folklore du Languedoc (Gard - Hérault - Lozère), Paris, G.-P. Maisonneuve, 1960, p. 128 «Cérémonie de l'oignon»; repris dans Promenade à travers les traditions populaires languedociennes, des Cévennes à la mer, Paris, Maisonneuve et Larose, 2001, p. 128. Référence fournie par Francine Brunel-Reeves.

${ }^{46}$ "Le Mariage de la cadette», dans Revue de folklore français, Paris, Librairie Larose, tome V, ${ }^{\text {os }} 4-5$, juillet-octobre 1934, p. 305-306. 
ciant l'aînée célibataire au four, la situation était tout autre un siècle plus tôt dans le nord de la France et en Belgique, où cette sanction verbale accompagnait une coutume bien répandue. Déjà en Wallonie, l'expression aurait été liée à une danse rituelle existant sans doute à une époque plus ancienne. C'est Eugène Monseur qui signale ces locutions dans trois provinces belges: dans le Brabant (à Nivelles), le Luxembourg (à Laroche) et le Hainaut, on disait «d'une jeune fille qui se marie avant sa sœur aînée [...] : Elle fait danser sa sœur sur le cul du four ${ }^{47}$ ". Ces traditions seraient de nos jours inexistantes selon l'avis de Roger Pinon, folkloriste wallon ${ }^{48}$. Ailleurs, en Flandre française, l'expression est associée à un rituel en 1934:

La phrase: "On va vous faire monter sur le four», adressée à la jeune fille dont la sœur plus jeune se marie avant elle, n'est plus aujourd'hui qu'une formule non suivie d'exécution. Il n'en était pas de même jadis. Un exemple m'en a été fourni par $\mathrm{M}^{\text {me }}$ Devoddère-Charlet, originaire de Wavrin (arr. de Lille, Nord). «Il y a une soixantaine d'années [vers 1874], à Wavrin, les jeunes gens invités au mariage de $\mathrm{M}^{\mathrm{lle}} \mathrm{P}$..., d'une bonne famille du pays, s'amusèrent à porter effectivement sur le four la sœur aînée de la mariée. $\mathrm{M}^{\mathrm{me}} \mathrm{D}$...-C... tient le fait de sa mère, qui était parmi les invitées ${ }^{49}$ ".

Selon $\mathrm{M}^{\text {lle }} \mathrm{C}$. Leroy, cette sanction s'appliquait aussi aux jeunes hommes célibataires dans le Pas-de-Calais ${ }^{50}$. Pour sa part, P. Saintyves notait: «Le groupe social n'entend pas que parmi ses membres il y en ait qui se permettent d'échapper au devoir de procréer. Mais il ne se contente

${ }^{47}$ Eugène Monseur, Le Folklore wallon, Bruxelles, Charles Rozez, libraireéditeur, [1892], p. 36: "èl fé dansé s'syeûr su l'ku du foûr (Nivelles)» et à Laroche (Luxembourg) «Elle fait danser sa sœur sur le cul du four »; i-l va fé dansé s'soûr so l'kou do for "elle va faire, etc.». Id., "Coutumes. I. - Les Noces", dans Bulletin du folklore wallon, Liège et Bruxelles, vol. III, tome 2, $1^{\text {er }}$ fascicule, janvier-mars 1893, p. 25.

${ }^{48}$ Roger Pinon, lettre du 14 juillet 1999: «Je ne connais en Wallonie, autrefois ou de nos jours, un rituel du mariage de la cadette avant l'aînée."

${ }^{49}$ Revue de folklore français, Paris, Librairie Larose, tome V, $\mathrm{n}^{\mathrm{os}} 4-5$, juilletoctobre 1934, p. 305-306: "Le Mariage de la cadette".

${ }^{50}$ Ibid. : observation du chanoine Fournier, région de Fruges (arr. de Montreuil). 
pas de railler et de satiriser, il s'efforce de préparer le réticent à remplir son devoir. Ainsi faisait-on jadis en le transportant sur le cul du four ${ }^{51} »$.

De même, Arnold Van Gennep consigne ces faits pour le nord et l'est de la France. D'abord, dans la région de SaintOmer, "aux débuts du XIX ${ }^{\mathrm{e}}$ siècle», «il fallait que dans quelque moment de la fête [l'aînée] fût saisie, enlevée et portée sur la voûte du four, afin, disait-on, qu'elle s'échauffât, puisqu'il semblait résulter de sa position qu'elle était restée insensible à l'amour ${ }^{52}$ ». Dans la même région, à Wizennes, il remarque que "le dépôt sur le four a été remplacé par une promenade en brouette et, dans quelques villages de la région de La Bassée, a été établi un lien entre le dépôt sur le four et l'offre d'un morceau de tarte retournée ${ }^{53} »$.

\section{La cuve}

Un rituel plutôt marginal, qui comme le transport sur la voûte du four a donné naissance à une expression populaire, mérite qu'on le rapporte ici. Ce trait intéressant a été noté par Van Gennep à propos de la locution "elle l'a mis "sous la tinette" " relevée en Champagne et qui, à SainteMenehould, « est dramatisée au cours du repas : deux vieux garçons apportent une petite cuve qu'ils tiennent audessus de l'aîné pendant quelque temps ${ }^{54} »$. Cette mise en scène n'est pas sans rappeler l'exhibition de la cuve qui précède la danse de l'aîné célibataire dans le nord de l'Ontario.

\footnotetext{
${ }^{51}$ Loc. cit.

${ }^{52}$ Van Gennep, op. cit., tome I, vol. 2, p. 632. C'est l'interprétation que retient Roger Pinon, dans sa lettre du 14 juillet 1999: «Il faut comprendre que danser sur le cul du four est un fait de langage pour exprimer qu'il faut réchauffer l'aînée et son ardeur amoureuse, vu qu'elle s'est laissée devancer par sa sœur.

${ }^{53}$ Ibid., p. 633.

${ }^{54}$ Ibid., p. 629-630.
} 


\section{La chèvre blanche}

En Lorraine, Auricoste de Lazargue a signalé une singulière coutume où intervient une chèvre blanche. Cette pratique est remarquable parce que «le marié ou la mariée doit acheter une chèvre à son frère ou à sa sœur» qui est son aîné ${ }^{55}$. L'auteur s'empresse d'ajouter que, vers 1870 dans le pays messin où il fait ses observations, on n'achetait plus vraiment de chèvre et qu'on pouvait simplement l'emprunter pour la durée de la prestation si on n'en possédait pas. Le lendemain des noces, pendant le repas du midi qui avait lieu au domicile de la mariée, "on amène une chèvre enrubannée et portant un chou attaché à ses cornes. Le marié ou la mariée, suivant le cas, offre la chèvre à son frère ou à sa sœur et celui-ci ou celle-là se lève, prend la bête par la corde et l'emmène ${ }^{56} »$. Un autre témoin rapporte même l'exclamation qui en consacrait l'usage: "Vol lè chive qu'ot lièye! (Voilà la chèvre qui est liée) ${ }^{57}$ ". Gabriel Jeanton décrit le don de la chèvre comme une tradition courante en Bourgogne, dans le Mâconnais; il note que l'aîné, garçon ou fille, «se promenait pendant toute la noce et notamment pendant le bal des soupes, en sabots et avec une chèvre blanche qui lui était offerte par l'épousée ${ }^{58} »$. Ce rituel serait plus ancien encore d'après Arnold Van Gennep qui en note ainsi l'évolution:

\footnotetext{
${ }^{55}$ E. Auricoste de Lazargue, "Coutumes de mariage", dans Revue des traditions populaires, vol. 24, $\mathrm{n}^{\circ} 7$, juillet 1909, p. 226.

${ }^{56}$ Ibid, p. 227.

${ }^{57}$ Revue de folklore français, Paris, Librairie Larose, tome V, $\mathrm{n}^{\mathrm{os}} 4-5$, juilletoctobre 1934, p. 305-306: "Le Mariage de la cadette»: selon le $\mathrm{D}^{\mathrm{r}}$ de Westphalen : Petit Dictionnaire des traditions populaires du pays messin, Metz, 1934, Vo Chèvre, col. 95.

${ }^{58}$ Gabriel Jeanton, Le Mâconnais traditionaliste et populaire. Fêtes du terroir et coutumes du foyer, Macon, Protat frères, imprimeurs, 1922, vol. 4, p. 50-51; Id., Revue de folklore français, Paris, Librairie Larose, tome V, $\mathrm{n}^{\mathrm{os}} 4-5$, juilletoctobre 1934, p. 305-306: «Le Mariage de la cadette». Van Gennep signale ce rituel comme la «Danse spéciale de la fille aînée non mariée, en sabots et avec une chèvre", op.cit., tome I, vol. 2, p. 546.
} 
Dans l'arrondissement de Remiremont (Vosges), à la fin du XVIII ${ }^{e}$ siècle et jusque vers la fin du $\mathrm{XIX}^{\mathrm{e}}$, dans la plupart des villages, la mariée devait faire cadeau à son aînée d'une chèvre blanche enrubannée; peu à peu, cependant, cette chèvre vivante a été remplacée par une chèvre en bois ou en carton, simple jouet, ou même taillée dans un navet, d'où l'expression «emmener la bique»; parfois, au lieu d'une chèvre blanche, la mariée donnait un mouton [aussi à l'aîné, dans tout le pays messin; la chèvre était parfois empruntée], elle doit être enrubannée et ornée de fleurs; par endroits, on fixe un chou à ses cornes [Nancy, Moselle et Moselle luxembourgeoise]. Cette zone lorraine semble se continuer sporadiquement en Champagne... [...] En Savoie, j'ai trois points éloignés: à Thonon, où le frère ou le cousin de l'aînée lui amenaient une chèvre qu'elle caressait, à laquelle elle donnait à manger et dont on bandait ensuite les yeux avec la jarretière de la mariée; à Val d'Isère... à Fontcouverte (Maurienne)... Enfin, dans le nord de la Touraine, on amène à la table des mariés une chèvre enrubannée et le garçon d'honneur [sans doute l'aîné] lui donne une poignée de choux ${ }^{59}$.

Cette coutume se pratiquait toujours en 1984 dans la région de Rochesson, selon l'informateur jurassien de Christine Amiel ${ }^{60}$ :

Si un garçon ou une fille se marie avant son frère ou sa sœur aîné il doit lui offrir une chèvre. Moi, je me suis marié avant mon frère, alors le jour de la noce je lui ai offert une chèvre en bois. On l'avait recouverte de coton pour faire la laine et pour qu'elle soit blanche. Souvent on fait aussi avec une chèvre en peluche; mais un de mes cousins m'a dit qu'une fois il avait vu donner à l'aîné deux chèvres chamoises vivantes ${ }^{61}$.

Offerte à l'aînée, «elle pourrait, comme le voudrait Saintyves, rappeler une coutume de bestialité, par remplacement dit obscène ${ }^{62}$ ", mais on l'offre parfois à la cadette également. Cherchant alors les raisons de la présence de la chèvre dans cette tradition, Christine Amiel scrute surtout

\footnotetext{
${ }^{59}$ Van Gennep, op. cit., tome I, vol. 2, p. 633-634.

${ }^{60}$ Christine Amiel, "Les Caprices du mariage», dans Ethnologie française, Paris, Société d'ethnologie française, Nouvelle série, tome 17, $\mathrm{n}^{\circ} 4,1987$, p. [367]-380. Référence de Martine Duquesne, courriel du 26 mars 2003.

${ }^{61}$ Ibid., p. [367].

${ }^{62}$ Van Gennep, op. cit., tome I, vol. 2, p. 634.
} 
la nature même de cet animal qui se caractérise par son insoumission, son inconstance et son ambivalence sexuelle; car on lui doit le mot caprice ${ }^{63}$ : «Qu'elle nomme et dénonce la jeune effrontée qui va au devant des garçons, la femme qui dérobe l'autorité à l'homme, l'amoureuse trop chaude ou trop froide, la vieille à la féminité révolue, l'adolescente encore "garçonne", la chèvre incarne toujours ici les multiples limites de la féminité ${ }^{64} »$. Aussi serait-elle «en mesure de signifier toutes les étapes, toutes les positions féminines, de stigmatiser tous les désordres de l'amour ${ }^{65}$ ». Mais en plus de "dénoncer une anormalité ", elle est capable de « la résoudre »: "Car en étant porteuse des traits antagonistes de la stérilité et de la fécondité[,] la chèvre apparaît douée du pouvoir de faire passer les femmes d'un état à l'autre ${ }^{66} »$. Cette association constituerait en quelque sorte un appel à l'aide qu'on ferait symboliquement lancer par l'aînée à cet animal, divinisé par les rubans, les ornements floraux et autres soins dont on l'honore, pour la tirer de son mauvais pas; la jarretière de la mariée, qui laisse habituellement présager le mariage de celui qui l'attrape dans d'autres rituels et avec laquelle on bande les yeux de la chèvre dans un cas, autoriserait peut-être quelque acte divinatoire à l'instar de bons augures. Mais il vaut mieux admettre que, donnée ainsi en spectacle, l'aînée avait, comme dans la danse rituelle au Canada français, de plus fortes chances d'attirer les regards des prétendants disponibles.

\section{Comparaison}

Comme l'Amérique française, la France possédait aussi des expressions riches et encore plus variées, évoquant tantôt le renversement de la normalité, tantôt la mise à

\footnotetext{
${ }^{63}$ Amiel, op. cit., p. 368.

${ }^{64}$ Ibid., p. 372.

${ }^{65}$ Ibid., p. 373.

${ }^{66}$ Ibid., p. 376.
} 
l'écart ou, parfois, le rejet des aînés qui avaient dérogé à leur devoir d'exemplarité et omis de «donner le bon exemple» à leurs cadets en ne se mariant pas les premiers. Là non plus, on ne les fustigeait pas seulement en paroles, mais on les soumettait à des rituels publics et «exemplaires» pour s'en amuser le jour du mariage de leur cadet: porter un sac d'avoine ou les hautons, ou un tribart, filer la quenouille, manger l'oignon ou la salade, être porté sur le cul du four, conduire une chèvre blanche furent en leur temps les formes les plus populaires, en tout cas les mieux attestées. Ces rites, élaborés aux mêmes fins que leurs parallèles nord-américains, incorporent cependant peu des éléments présents chez ces derniers et, le cas échéant, dans des arrangements fort différents: l'expression «manger de l'avoine» est universelle chez nous, mais le port d'un sachet d'avoine est marginal et n'a été relevé que dans une famille franco-manitobaine d'origine belge ${ }^{67}$; de même, la cuve, qui survit aussi en France dans l'expression "décuveler la lessive", maintenue quelques instants au-dessus de la tête de l'aîné ressemble au geste qui précède l'installation et la danse dans la cuve en Ontario français; enfin, les rituels liés au tribart, à la quenouille, à l'oignon, au cul du four et à la chèvre blanche sont totalement inconnus chez nous ${ }^{68}$. Bien plus étonnant encore, l'élément le plus courant en Amérique, la danse, cadre essentiel qui colore toutes les formes de nos rituels, ne figure en France que sporadiquement: «danser avec les hautons", «danser sur le cul du four " et la "danse spéciale de la fille aînée non mariée, en sabots et avec une chèvre", notée en Bourgogne à l'occasion du bal des noces ${ }^{69}$, en seraient les plus proches parents. Aucune attestation fran-

${ }^{67} \mathrm{Cf}$. notre article «"Danser sur ses bas". Rémanence d'un rituel du mariage franco-ontarien», op. cit., p. 308 (et la note 148).

${ }^{68}$ L'association de la chèvre à un rituel initiatique existe aussi au Canada français, l'expression «sauter la chèvre " étant le nom donné au rite d'initiation des Chevaliers de Colomb.

${ }^{69}$ Van Gennep, op. cit., p. 546. 
çaise, donc, de la danse de l'aîné nu-pieds ou en pieds de bas, dans une auge ou dans une cuve. Comment expliquer l'absence contemporaine de cette forme de la tradition en France, elle qui aurait dû en être le foyer de diffusion pour la francophonie nord-américaine? Les données de terrain ne manquent pourtant pas. Cette forme particulière auraitelle échappé aux investigations des enquêteurs? L'auraiton bien involontairement négligée ou serait-elle sortie de la tradition avant les premières enquêtes? Cette dernière possibilité est sans doute la bonne, car, à l'exception de la chèvre blanche, tous ces rituels semblent aujourd'hui complètement délaissés en France. Déjà, en 1846, une George Sand décrivant une noce campagnarde, «à laquelle [elle] eu[t] le plaisir d'assister [...] quelques années » auparavant, n'en trouve pas trace dans le Berry, une province « stationnaire » qu'elle juge « après la Bretagne et quelques provinces de l'extrême midi de la France, [...] le pays le plus conservé qui se puisse trouv[er] à l'heure qu'il est ${ }^{70} »$. Un ethnologue contemporain, Martine Segalen, "[c]ombinant les informations des manuels des folkloristes et les témoignages recueillis sur le terrain" afin d'étudier "le folklore du mariage à la fin du XIX ${ }^{\mathrm{e}}$, et celui d'aujourd'hui» ne fait aucune allusion non plus à notre rituel $^{71}$. Des réponses orales et écrites de nombreux collègues et de témoins de la Bourgogne, de l'île-de-France, du Poitou, de la Bretagne et d'ailleurs ne nous ont apporté aucune trace vivante de cette sanction du mariage. Le même silence vaut pour la Belgique, tant wallonne que flamande, et pour la Suisse romande.

\footnotetext{
${ }^{70}$ George Sand (1804-1876), La Mare au diable. Chronologie et préface par Pierre Reboul, [Paris], Garnier-Flammarion, «GF-Texte intégral » n 35,1964, p. 153.

${ }^{71}$ Martine Segalen, Nuptialité et alliance. Le choix du conjoint dans une commune de l'Eure, Paris, G.-P. Maisonneuve et Larose, « Mémoires d'anthropologie française", 1972, p. 111-120; ce rituel est aussi absent de son plus récent livre: Éloge du mariage, [Paris], Découvertes Gallimard, coll. «Culture et société », [2003], 128 p.
} 
À moins que sa source ne soit ailleurs... C'est à cette dernière option qu'il faudrait conclure si l'on s'en tenait strictement à la remarque de Van Gennep qui renvoie à l'Angleterre et à l'Écosse ces "pénalités ridicules, de danser pieds nus ou de porter des jarretières vertes ${ }^{72} »$.

\section{III - UNE TRADITION CELTIQUE}

\section{A. LA FILIĖRE IRLANDAISE ET BRITANNIQUE}

Cette voie mérite d'être sérieusement explorée, notamment du côté de l'Irlande depuis que Paul Delarue, le grand spécialiste français du conte populaire, l'a désignée comme le foyer de diffusion de formes et de motifs canadiens particuliers. Ce dernier n'ignorait pas les enquêtes que menaient en Amérique française les Barbeau, Carrière, Lacourcière, Savard et Roy, et il en rendit tout spécialement compte dans une note bien informée qui allait trouver ici des échos pendant un demi-siècle ${ }^{73}$. La lecture experte qu'il fit de la vingtaine de collections recensées l'amena à cette remarque qui entame son compte rendu: «Si la majorité des contes du Canada de langue française vient de chez nous, un nombre qui n'est pas négligeable est de provenance irlandaise». Il avait reconnu que certains récits de ces "collections canadiennes" présentaient «une forme qui ne se trouve pas chez nous, mais qui, en revanche, correspond d'une manière frappante aux formes notées en Irlande, et même à [...] des versions typiquement irlandaises ». Et, dans l'ouvrage de sœur Marie-Ursule qu'il recensait parmi d'autres, il en découvrit sans peine une justification: «On s'explique cette pénétration par le grand nombre d'Irlandais immigrants et par la facilité avec laquelle l'élément irlandais qui s'est fixé dans le pays a été assimilé par l'élément français à la suite de mariages

\footnotetext{
${ }^{72}$ Van Gennep, op. cit., p. 635: il se réfère alors à sa traduction du livre d'Edward Westermarck, Histoire du mariage, tome II, p. 111-114.

${ }^{73}$ Paul Delarue, "Collections de contes canadiens", dans Arts et traditions populaires, vol. 1, 1953, p. 276-282.
} 
mixtes $^{74} »$. Cette interprétation ne doit cependant pas voiler l'assurance de l'enquêteuse qui, commentant la décroissance de l'élément irlandais par de nombreux départs et un taux d'assimilation effarant, déclare qu' «aujourd'hui, à l'exception des quelques familles tout à fait irlandaises, ils [les Lavalois] ne forment qu'une nationalité française ${ }^{75} »$; ce que sa conclusion renforce encore: «si l'influence irlandaise fut considérable au début, elle est allée en diminuant de telle sorte qu'elle est à peine perceptible. Sauf le blason populaire et quelques traditions déjà citées, il n'existe plus, à Sainte-Brigitte-de-Laval, de véritable folklore irlandais ${ }^{76} \%$.

Quelques années plus tard, dans le bilan qu'il dressait des recherches sur «le Conte populaire français en Amérique du Nord», Luc Lacourcière avait bien enregistré la leçon de Delarue: "L'examen attentif des contes canadiens, quant à leur provenance, nous réserve plusieurs surprises", annonçait-il avant d'endosser avec une certaine retenue la position de son maître français: s'il adhérait de prime abord à cette « filiation irlandaise et écossaise, qui devrait être examinée de plus près ", c'est qu'elle semblait aller de soi en raison des nombreux rapports entre ces groupes «par les mariages et surtout par les travaux en commun dans les chantiers forestiers". Néanmoins, une chose le chicotait: «c'est que cette filiation n'ait apparemment joué que dans le sens de l'assimilation française", puisqu' «un nombre infime de contes en langue anglaise, moins d'une cinquantaine, ont été recueillis à travers tout le Canada, ou en gaélique, au Cap-Breton ${ }^{77} »$. Dans les

\footnotetext{
${ }^{74}$ Ibid., p. 278.

${ }^{75}$ Sœur Marie-Ursule, Civilisation traditionnelle des Lavalois. Préface de Luc Lacourcière, Québec, PUL, "Archives de folklore», 5-6, 1951, p. 36; en fait, selon le tableau de la page 32, parmi les 962 habitants recensés en 1941, 18 sont des Irlandais, soit $1,8 \%$.

${ }^{76}$ Ibid., p. 360.

${ }^{77}$ Luc Lacourcière, "Le Conte populaire français en Amérique du Nord", dans Internationaler Kongreß der Volkserzählungsforscher in Kiel und Kopenhagen (19.8.-29.8. 1959), Berlin, de Gruyter, 1961, p. 149-150.
} 
années suivantes, deux études comparées, préparées sous la direction de Lacourcière lui-même, ont identifié une influence celtique dans la transmission du conte populaire au Canada français et leurs auteurs désignèrent l'Irlande comme responsable de cette diffusion ${ }^{78}$.

\section{La Grande-Bretagne \\ a. Danser pieds nus au temps de Shakespeare ${ }^{79}$}

La Grande-Bretagne possède des attestations anciennes de la sanction populaire à l'étude, dans des formes très voisines des nôtres, et même la plus ancienne de toutes. William Shakespeare (1564-1616) fournit cette toute première mention dans une comédie, La Mégère apprivoisée, dont la composition remonte à $1594^{80}$. Cette pièce porte précisément sur le respect de l'ordre de la naissance dans le mariage, car Baptista Minola, le père, refuse de marier sa cadette Bianca tant que Catharina, l'aînée et la mégère, n'aura pas trouvé un époux: "BAPTISTA. - Messieurs, ne m'importunez pas davantage; vous connaissez ma ferme résolution de ne pas donner ma cadette avant d'avoir un mari pour l'aînée » (Acte I, scène I). En attendant, Bianca, qui a plusieurs prétendants, s'adonnera à l'étude de la musique et de la philosophie. Survient alors Petruchio, un original, qui offre à Baptista d'épouser la farouche Catharina, car la dot est bonne; répondant aux injures de la mégère par des compliments et des flatteries, il fixe le mariage au dimanche suivant. Arrivé en retard à cette

\footnotetext{
${ }^{78}$ Hélène Bernier, La Fille aux mains coupées (conte-type 706), Québec, PUL, "Archives de folklore», 12, 1971, XII-191 p.; et Nancy Schmitz, La Mensongère (conte-type 710), Québec, PUL, «Archives de folklore», 14, 1972, XIV-310 p.

${ }^{79}$ Cette section, qui répond à la division «Danser en semelles de bas au temps des Papineau" de notre premier article, Cahiers Charlevoix 5, p. 246-250, justifie aussi le titre de cette deuxième étude.

${ }^{80}$ William Shakespeare, Les Deux Gentilshommes de Vérone, La Mégère apprivoisée, Peines d'amour perdues. Traduction de François-Victor Hugo. Préface et notices par Germaine Landré, [Paris], Garnier-Flammarion, [ «GF/Texte intégral» 47], [1965], 313 p. Cette pièce comprend cinq actes précédés d'un prologue.
} 
cérémonie, le nouveau marié humilie Catharina par son costume de bouffon et son attitude désobligeante avant de l'emmener de force chez lui, laissant les invités en plan. Catharina est privée de sommeil, de nourriture et même d'un beau costume parce que Petruchio les juge indignes d'elle. En la ramenant chez son père, Petruchio la met à l'épreuve et la persuade que le soleil est la lune et qu'un vieillard rencontré est une dame. Prétendant que sa femme est la plus docile, il mettra ensuite au défi les nouveaux maris, celui de Bianca et un ancien prétendant: seule, Catharina répondra sur le champ à la demande de Petruchio et donnera ainsi une leçon de soumission aux autres épouses.

L'allusion à la sanction de l'aînée se situe au deuxième acte, au moment où le drame se noue, lorsque Catharina, l'aînée célibataire et la mégère, crie à son père devant l'imminence du mariage de Bianca, sa sœur cadette: "Catharina. - Je le vois à présent, c'est elle qui est votre trésor et il faut qu'elle ait un mari; moi, il faut que je danse pieds nus le jour de sa noce, et que, pour l'amour d'elle, je mène des singes en enfer ${ }^{81}$ " (Acte II, scène I). Malheureusement, pas plus que dans la tradition canadienne relayée par Joseph Papineau en 1826, on ne saura jamais comment se déroulait la sanction shakespearienne, puisque la mégère, convolant le dimanche suivant, n'eut jamais à danser. Aucune didascalie non plus ni autre note de l'écrivain qui renseignerait le lecteur sur la nature du châtiment imposé à l'aînée; cette omission permet de supposer que le public contemporain était bien au fait de la coutume, sans quoi la menace qui met la mégère aux abois aurait été

${ }^{81}$ Ibid., acte II, scène I, vers 31-34, p. 141. Pour la version originale: William Shakespeare, The Taming of the Shrew, dans The Comedies. The Histories. The Cambridge text established by John Dover Wilson for the Cambridge University Press, London, Octopus Books Ltd, 1986, c1921, Act 2, Scene I (1. 31-34), p. 252: «KATHARINA. What, will you not suffer me? Nay, now I see/She is your treasure, she must have a husband,/I must dance bare-foot on her weddingday/And for your love to her lead apes in hell». 
incomprise et aurait fait obstacle à l'intelligence des enjeux de la comédie. Ici encore, la tradition franco-américaine aplanit l'interprétation du premier élément de cette plainte, soit l'obligation, pour l'aînée qui n'a pas trouvé chaussure à son pied, de danser pieds nus. Le second élément, la conduite de singes en enfer, resterait obscure sans les commentaires qui accompagnent les traductions: les vieilles filles, qui refusent de donner naissance à des enfants et ainsi de les mener en paradis, seraient, selon l'usage populaire du XVI ${ }^{\mathrm{e}}$ siècle, tournées en dérision et condamnées à mener des singes aux enfers ${ }^{82}$.

Un trait additionnel mérite considération. Quand, au troisième acte (scène II), Petruchio se présente en costume ridicule le jour de son mariage, son valet porte également une tenue comparable à la sienne; le témoin qui la décrit, " avec un bas de fil à une jambe et une chausse de grosse laine à l'autre, jarreté d'un cordon rouge et d'un bleu", la juge inconvenante et inhabituelle tellement elle «ne ressembl[e] en rien au page d'un chrétien ou au laquais d'un gentilhomme». Est-il possible que cet accoutrement, saugrenu par ses pièces discordantes et ses jarretières de couleurs incompatibles, ait quelque chose à voir avec les chaussettes dépareillées de la danse franco-ontarienne?

Cette représentation inopinée, apparue sous la plume du dramaturge anglais, s'estompera pendant près de deux siècles, voilant la marche de la tradition. Elle reparaîtra en

\footnotetext{
${ }^{82}$ Voir les commentaires dans les traductions suivantes: La Sauvage apprivoisée, dans CEuvres complètes. Avant-propos d'André Gide. Introduction générale et textes de présentation d'Henri Fluchère, [Paris, Librairie Gallimard], NRF, «Bibliothèque de la Pléiade», [1959], vol. I, p. 976-977 et p. 1417; La Mégère apprivoisée, dans Théâtre complet. Traduction de François-Victor Hugo. Introduction, notices et notes par J[oseph]-B[arthélémy] Fort, Paris, Éditions Garnier Frères, 1961, tome I, p. 613; La Mégère domptée, dans Les Comédies. Nouvelle traduction française par Pierre Messiaen. Introductions et commentaires par Michèle Bernard, Félix Carrère, Solange Dayras, Françoise Gutel, J.-P. Hulin et Patrick Rafroidi, sous la direction de José Axelrad, [Paris], Desclées de Brouwer, [1961], p. 828.
} 
un tableau à peu près inchangé en 1787 parmi les superstitions consignées dans le glossaire de Francis Grose: « $\mathrm{Si}$ la plus jeune fille d'une famille se marie par hasard avant ses sœurs aînées, elles doivent toutes danser à sa noce les pieds nus. Ceci, dit-on, détruit la mauvaise chance et leur procure des maris ${ }^{83} »$.

\section{b. Danser pieds nus ou en semelles de bas au XIX $\mathrm{X}^{e}$ siècle}

La tradition de danser pieds nus, en semelles de bas ou sans chaussures a survécu en Grande-Bretagne jusqu'à la fin du XIX ${ }^{\mathrm{e}}$ siècle, comme il est avéré par les travaux de quelques compilateurs. Parmi ceux-ci, Charlotte Sophia Burne mentionne que, dans le Shropshire, une fille aînée dut danser en semelles de bas, "in stocking-feet», au mariage de sa cadette à Hodnet en 1881. Mais cet auteur raconte aussi un fait cocasse survenu la même année: une tante intransigeante, furieuse qu'une pratique aussi obligatoire à son époque ait été négligée par la jeune génération, semonça vertement sa nièce rencontrée à Wellington le lendemain des noces: "Ainsi j'apprends que tu n'as pas dansé pieds nus ! J'ai honte de toi! Si j'avais été là, je te l'aurais fait faire. J'ai envie de t'enlever tes chaussures maintenant et de te faire danser dans la rue $^{84} »$. Les relevés d'Edward

${ }^{83}$ Francis Grose (1731-1791), A Provincial Glossary, with a Collection of Local Proverbs and Popular Superstitions, [London, S. Hooper, 1787], Menston, The Scholar Press, «A Scholar Press Facsimile», 1968, p. 62 [Superstitions]. Cette traduction littérale est de Van Gennep et extraite du livre d'Edward Westermarck (1862-1939) qui, au deuxième tome de son Histoire du mariage (II L'attraction sexuelle. La jalousie masculine. Traduit de l'anglais par Arnold Van Gennep. Troisième édition, Paris, Mercure de France, 1935, p. 113. L'édition originale de cet ouvrage date de 1891 : The History of Human Marriage, London et New York, Macmillan, 1891, xix-644 p.), cite cette même source d'après un document découvert chez John Brand (1744-1806), Observations on Popular Antiquities: including the whole of Mr. Bourne's Antiquitates Vulgares, with addenda to every chapter of that work: as also, an appendix, containing such articles on that subject, as have been omitted by that author, Newcastle upon Tyne, By T. Saint, for J. Johnson, London, 1777, p. 398.

${ }^{84}$ Charlotte Sophia Burne, Shropshire Folklore: A Sheaf of Gleanings from the collections of Georgina F. Jackson, London, Trübner,1883, p. 290-291. Traduction de l'auteur. 
Westermarck montrent que la pratique était également répandue en plusieurs autres régions de la Grande-Bretagne avec des variantes locales: au pays de Galles, «si le cadet d'une famille se mariait avant ses aînés, ceux-ci devaient danser sans souliers comme pénitence vis-à-vis de l'assemblée ${ }^{85}$ "; William Henderson consigne la même expression en 1866 dans le comté de Durham, au nord de l'Angleterre, sans confirmer si le rituel a toujours lieu ${ }^{86}$. Dans son étude des mariages anglais du XVII ${ }^{e}$ siècle à nos jours, John Gillis reprend pour l'essentiel ces mêmes sources auxquelles il ajoute quelques parallèles ${ }^{87}$. Contrairement à la plupart des exemples britanniques qui visent les filles célibataires, l'un d'eux signale le cas où, dans la vallée de Clwyd au pays de Galles, un garçon dansa "sans chaussures» le jour des noces: le témoin, une femme de 50 à 60 ans, née au début du XIX ${ }^{e}$ siècle, expliqua que, le jour de son mariage, «son frère avait subi cette punition pour l'avoir laissée, elle, sa cadette, se marier avant lui ${ }^{88} »$. Dans le Devonshire, à l'ouest de l'Angleterre, on dit que l'aînée dansait dans des bas verts, «in green stockings $»^{89}$.

À la même époque, une correspondance unique entre deux lecteurs prit place dans les colonnes du périodique Notes and Queries, entre le 25 août 1883 et le 26 juillet 1884. À une interrogation d'Alpha, qui s'enquiert si la

${ }^{85}$ Westermarck citant Marie Trevelyan, Folk-Lore and Folk-Stories of Wales, Introduction by E. Sidney Hartland, London, Elliot Stock, 1909, p. 274.

${ }^{86}$ William Henderson, Notes on the Folk-Lore of the Northern Counties of England and the Borders, [London, 1866; London, The Folk-Lore Society, 1879], Nendeln, Kraus Reprint, 1967, p. 41.

${ }^{87}$ John R. Gillis, For Better, For Worse. British Marriages, 1600 to the Present, New-York et Oxford, Oxford University Press, 1985, p. 72.

${ }^{88}$ Elias Owen, Old Stone Crosses of the Vale of Clwyd and Neighbouring Parishes together with some account of the ancient manners and customs and legendary lore connected with the parishes, London, Bernard Quaritch, [1886], p. 62. Référence signalée par Gillis, loc. cit.

89 "Twenty-Seventh report on Devonshire Folk-Lore», dans Reports and Transactions, Plymouth, Devonshire Association for the Advancement of Science, Literature and Art, vol. 57, 1925, p. 128: le commentateur cite une source de 1862. 
pénitence de l'aînée célibataire forcée de porter un balai ${ }^{90}$ le jour du mariage de sa sœur, qu'il tient d'un cousin, est connue ailleurs que dans le Gloucestershire, et qui rajoute, à la livraison suivante, qu'on vient de lui communiquer un cas où l'aînée, dans le Worcestershire, doit danser nupieds ou sauter par-dessus une auge à cochon, William Deane répond qu'une vieille résidente de Suffolk l'a informé que l'aînée devait plutôt danser dans une auge à cochon. Cet échange éclairant, paru sous le titre «A Wedding Custom ", révèle qu'au pays même de Shakespeare, le Worcestershire, la sanction de danser pieds nus avait survécu presque quatre siècles et que, dans ces circonstances, l'auteur de la Mégère apprivoisée n'aurait eu qu'à puiser dans le répertoire régional une coutume alors si courante que tout commentaire lui eût paru superflu. Mais il implique en outre que la danse rituelle de l'aînée célibataire, déjà en recul en Angleterre, avait été reléguée au rang de curiosité pour les chercheurs ${ }^{91}$.

\section{c. Danser dans une auge}

Cet échange a ainsi fait ressortir une deuxième forme du rituel, la danse dans l'auge. D'autres exemples sont également connus: en East-Anglia, selon une source, l'aînée dansait dans l'auge à cochon vers $1862^{92}$; selon une autre, un jeune homme célibataire de Bridgnorth dut, lui aussi,

\footnotetext{
${ }^{90}$ Mise en lien avec le mariage, que les fiancés des contes populaires contractent simplement en sautant au-dessus d'un balai, cette pratique devait le favoriser; Elias Owen, op. cit., p. 62-63, a trouvé au pays de Galles des témoins qui croyaient que ce rite avait réellement existé et qu'il était valide. La «danse du balai ", attestée souvent en France au bal des noces, ne semble avoir aucun rapport avec les célibataires: A. Van Gennep, op. cit., tome I, vol. 2, p. 545-550. ${ }^{91}$ Alpha, "A Wedding Custom», Notes and Queries, London, Oxford University Press, Published at the Office... by John C. Francis, Sixth Series, vol. 8, July-December 1883, [Aug. 25, '83], p. 147; Alpha, ibid., 6 ${ }^{\text {th }}$ Series, vol. 9, January-June 1884 [Feb. $16^{\text {'}} 84$ ], p. 135; William Deane, ibid., $6^{\text {th }}$ Series, vol. 9, January-June 1884 [April 19 '84], p. 315; Alpha, ibid., 6 ${ }^{\text {th }}$ Series, vol. 9, January-June 1884 [May 3, '84], p. 359; William Deane, ibid., $6^{\text {th }}$ Series, vol. 10, July-December 1884 [July 26, '84], p. 79.

92 "Twenty-Seventh report on Devonshire Folk-Lore», op. cit., p. 128.
} 
danser dans une auge à cochon au mariage de son cadet en 1881, tandis qu'à Suffolk, un couple d'aînés, un frère et sa sœur, accomplirent si bien ensemble leur danse rituelle dans une auge qu'à la fin le récipient ne résista pas et se fendit en deux ${ }^{93}$.

Après avoir considéré ces diverses formes rituelles, Gillis conclut: «Toutefois, la punition la plus commune était celle qu'on désignait par diverses appellations comme "la danse en chaussettes vertes" (dancing in green stockings), "la danse dans l'auge à cochons" (dancing the hogs trough), ou simplement "la danse en pieds de bas" (dancing in stocking (eet) $)^{94} »$.

\section{d. Porter des jarretières vertes}

En Écosse, cependant, la danse rituelle n'a jamais été signalée, mais, à la place, le port de jarretières vertes aurait joué un rôle identique: dans les environs de Balmoral, l'aînée "est obligée, même par la force, de porter des jarretières vertes "; à l'Est, «chez les mineurs de Fife, des jarretières vertes étaient accrochées en cachette aux vêtements du frère ou de la sœur aînés non encore mariés, puis, quand on les avait découvertes, liées au bras gauche et portées pendant toute la soirée ${ }^{95} \gg$. Dans une collection de proverbes écossais, de la collection Hislop publiée en 1862 , on trouve l'expression «elle leur a donné des bas verts", quand une jeune femme se marie avant ses sœurs aînées ${ }^{96}$, tandis que dans le nord-est de l'Écosse, Walter Gregor rapporte en 1881 que la cadette devait remettre à sa sœur aînée des jarretières vertes ${ }^{97}$. Le port d'un vêtement de cette couleur ne cesse d'étonner dans un pays où

\footnotetext{
${ }^{93}$ Charlotte Sophia Burne, op. cit., p. 291, pour ces deux exemples.

${ }^{94}$ Traduction de l'auteur du passage de John R. Gillis, op. cit., p. 72.

${ }^{95}$ Edward Westermarck, op. cit., p. 113-114.

${ }^{96}$ The Oxford Dictionary of English Proverbs, Oxford, 1975, p. 304. Référence de Fionnuala Williams.

${ }^{97}$ Walter Gregor (1827-1897), Notes on the Folk-Lore of the North-East of Scotland, [London, The Folk-Lore Society, 1881], Nendeln, Kraus Reprint,
} 
le vert était signe de malchance et ne devait en aucun cas être porté le jour du mariage ${ }^{98}$.

Les anecdotes du Shropshire exceptées, tous ces témoignages, déjà vieillots au moment où on les enregistre, n'auraient guère survécu après les années 1850 et les chercheurs n'ont trouvé que peu de choses pour la période contemporaine. Il se rencontre bien ici et là quelques signes de la persistance de ces coutumes: commentant l'expression "danser sur un fagot d'ajoncs ", relevée à propos de la fille aînée dans un comté du centre de l'Angleterre, le Northamptonshire, un rapporteur ajoute, selon un ouvrage de 1922, que le dicton est aussi connu dans le Dorsetshire, au sud-ouest, où la coutume se pratique encore parfois et qu'on y danse aussi sur ses bas ${ }^{99}$; de même, dans la région centrale des Midlands, on signale en 1930 que la danse de la fille aînée, sur les bas ou dans une auge à cochon, y aurait été pratiquée à une époque indéterminée ${ }^{100}$. Si la tradition n'est pas encore complètement éteinte du côté de l'archipel britannique, les experts consultés, des témoins et des chercheurs nous le laissent croire pour l'Angleterre ${ }^{101}$; il n'en va pas autrement pour l'Écosse: « Les danses et des choses comme ça sont inconnues chez nous. Je n'ai jamais vu rien de semblable, malheureusement", nous assure une autre collègue ${ }^{102}$.

1967, p. 90; citation reprise par Margaret Bennet, Scottish Customs from the Cradle to the Grave, Edinburgh, 1992, p. 124 : référence de Fionnuala Williams, courriel du 30 juin 1999.

${ }^{98}$ William Henderson, op. cit., p. 34-35.

${ }^{99}$ «Twenty-Seventh report on Devonshire Folk-Lore», op. cit., p. 128: «Wedding Dancing on a furze faggot».

${ }^{100}$ J[ames] Harvey Bloom, Folk-Lore, Old Customs and Superstitions in Shakespeare Land, London, Mitchell, Hughes \& Clarke, [1930], p. 15. Référence de Fionnuala Williams, courriel du 4 janvier 2000. Né en 1860, l'auteur a interrogé F.S. Potter à Crimscot (Cotswolds) et sa sœur centenaire (p. [iii]) sans doute plusieurs années avant cette publication.

${ }^{101}$ Entre autres, J.D.A. Widdowson, National Centre for English Cultural Tradition, Sheffield, courriel du 12 juillet 1999 communiqué par Janet Alton.

${ }^{102}$ Frances J. Fisher, School of Scottish Studies, University of Edinburgh, courriel du 2 août 1999. 


\section{L'Irlande}

Quant à l'Irlande, pays qui a été depuis longtemps le théâtre d'enquêtes intensives, cette tradition n'aurait même jamais été relevée. L'avis de Rionach ui Ogain de Dublin est, sur ce point, catégorique: "À ma connaissance, en Irlande, le rituel que vous décrivez dans votre lettre n'existe pas. Il est possible naturellement que ça, ou un rituel semblable de danse, existait ailleurs et que l'on ne l'a jamais documenté, mais ce n'est pas probable». Cette opinion autorisée est assortie d'un commentaire qui montre que le contexte favorisait pourtant ce type de sanction : «Il faut en même temps dire que le mariage fut ici très important d'ailleurs pour des raisons économiques, religieuses, pratiques et sociales. Alors ceux qui ne se mariaient pas étaient parfois des objets de ridicule ${ }^{103} »$. À l'appui, ce correspondant renvoie à une série de moqueries et de mystifications qui avaient cours en Irlande dans le but précis de fustiger la condition de célibataire. Contrairement aux autres rituels britanniques et européens qui se pratiquaient le jour des noces du cadet, le moment culminant de ces manifestations survenait au début du carême, après les jours gras, période qui était l'occasion de nombreux mariages. Bien qu'on ait aussi joué des tours aux célibataires le soir du mardi gras, ou qu'on leur ait jeté des cendres le mercredi des cendres ou lancé du sel le lendemain, la coutume la plus répandue fut le crayonnage du premier dimanche du carême, appelé Chalk Sunday. A leur retour de l'église, les célibataires étaient assaillis par des garçons et des filles, ou des jeunes gens surgissant de leurs cachettes, munis de morceaux de craie blanche ou colorée, qui n'avaient de cesse de marquer les vêtements de leurs victimes de rayures ou de signes divers. Ces pratiques, très populaires au milieu du $\mathrm{XIX}^{\mathrm{e}}$ siècle dans les provinces de

${ }^{103}$ Rionach ui Ogain, Department of Irish Folklore/Roinn Bhéaloideas Éireann, University College, Belfield, Dublin, lettre du 8 juillet 1999. 
Munster et de Leinster, tombèrent en désuétude peu avant la Deuxième Guerre mondiale ${ }^{104}$. Il y eut bien, le jour du mariage, un autre rituel destiné aux garçons célibataires et pour lequel on se servait d'un bas. Mais ce vêtement, lancé par la mariée à tous les célibataires masculins, jouait le même rôle que le bouquet de la mariée pour les filles: son but n'était pas de punir les aînés, mais d'encourager tous les célibataires présents en augurant que celui qui l'attraperait serait le prochain à se marier ${ }^{105}$. Il faut donc exclure la filière irlandaise : hors circuit dans ce cas-ci, elle n'aurait jamais pu constituer la courroie de transmission de cette sanction coutumière au Canada français.

${ }^{104}$ Kevin Danaher, "Chalk Sunday», dans The Year in Ireland, Cork, The Mercier Press, 1972, p. 47-48. Référence communiquée par Rionach ui Ogain. ${ }^{105}$ Précisions tirées des références que Fionnuala Williams nous a communiquées dans ses courriels du 27 janvier et du 18 février 2000: William Carleton, Traits and Stories of the Irish Peasantry, Buckinghamshire, Colin Smythe \& Maryland, Barnes \& Noble Books, 1990, vol. I [édition originale en 1844]; Samuel Lover, Rory O'More; A National Romance, Westminster, Archibald Constable \& Co., 1898, p. 439-440 [édition originale en 1837]. 


\section{TABLEAU 2}

Comparaison de la danse rituelle de l'aîné célibataire en Amérique française et en Grande-Bretagne

\begin{tabular}{|c|c|c|c|}
\hline \multirow[t]{2}{*}{ ÉLEMENTS } & \multirow[t]{2}{*}{ COMMUNS } & \multicolumn{2}{|c|}{ DIVERGENTS } \\
\hline & & AMÉRIQUE FRANCCAISE & GRANDE-BRETAGNE \\
\hline Aîné & $\begin{array}{l}\text { fille seulement } \\
\text { garçons et filles }\end{array}$ & $\begin{array}{l}\text { rare } \\
\text { toujours aujourd'hui } \\
\text { garcon seulement (auge sale) } \\
\text { autrefois }\end{array}$ & $\begin{array}{l}\text { le plus souvent } \\
\text { parfois } \\
\text { jamais }\end{array}$ \\
\hline Moment & jour de la noce & & \\
\hline $\begin{array}{l}\text { Danse } \\
\text { type de danse }\end{array}$ & $\begin{array}{l}\text { toujours } \\
\text { en solitaire }\end{array}$ & gigue & inconnu \\
\hline $\begin{array}{l}\text { Costume pieds nus } \\
\text { bas }\end{array}$ & $\begin{array}{l}\text { pieds nus } \\
\text { bas ordinaires } \\
\text { bas speciaux }\end{array}$ & $\begin{array}{l}\text { rare } \\
\text { fréquent autrefois } \\
\text { multicolores et ornés }\end{array}$ & $\begin{array}{l}\text { fréquent } \\
\text { parfois } \\
\text { bas verts parfois }\end{array}$ \\
\hline Autres pièces & & $\begin{array}{l}\text { rarement : } \\
\text { ceinture ou chapeau }\end{array}$ & $\begin{array}{l}\text { port de jarretières vertes } \\
\text { sans danse) } \\
\text { port d'un balai }\end{array}$ \\
\hline \multirow[b]{2}{*}{ ou subsimit } & à cochon & & \\
\hline & & $\begin{array}{l}\text { cuye, seau, bassin, banc, } \\
\text { boite, etc. }\end{array}$ & inconnue \\
\hline $\begin{array}{l}\text { propre } \\
\text { sale }\end{array}$ & & $\begin{array}{l}\text { parfois de nos jours } \\
\text { souvent autrefois }\end{array}$ & $\begin{array}{l}\text { non spécifiée } \\
\text { non spécifiée }\end{array}$ \\
\hline pour sauter & & rare & $\underset{\text { (par-dessus) }}{\text { rare }}$ \\
\hline $\begin{array}{l}\text { pour y boire } \\
\text { propre }\end{array}$ & & et danser autour & inconnue \\
\hline Jet de monnaie & & régulièrement & inconnu \\
\hline Durée & & de 5 à 45 minutes & inconnue \\
\hline Musique & & $\begin{array}{l}\text { rigodon } \\
\text { (volon ou orchestre) }\end{array}$ & inconnue \\
\hline Vitalité & & $\begin{array}{l}\text { auge : en déclin } \\
\text { bas : en pleine vigueur }\end{array}$ & $\begin{array}{l}\text { auge :éteinte (?) } \\
\text { bas : éteinte (?) }\end{array}$ \\
\hline
\end{tabular}

\section{Ailleurs}

Selon nos correspondants et les témoins interrogés, la danse rituelle de l'aîné célibataire n'est pas connue ailleurs en Europe, ni dans la péninsule ibérique (Espagne, Portugal), ni en Europe centrale (Allemagne, Hongrie, Italie, 
CARTE 2

AIRES DE CONCENTRATION ET FORMES

DE LA SANCTION RITUELLE DE L'AÎNÉ CÉLIBATAIRE

EN EUROPE DE L'OUEST

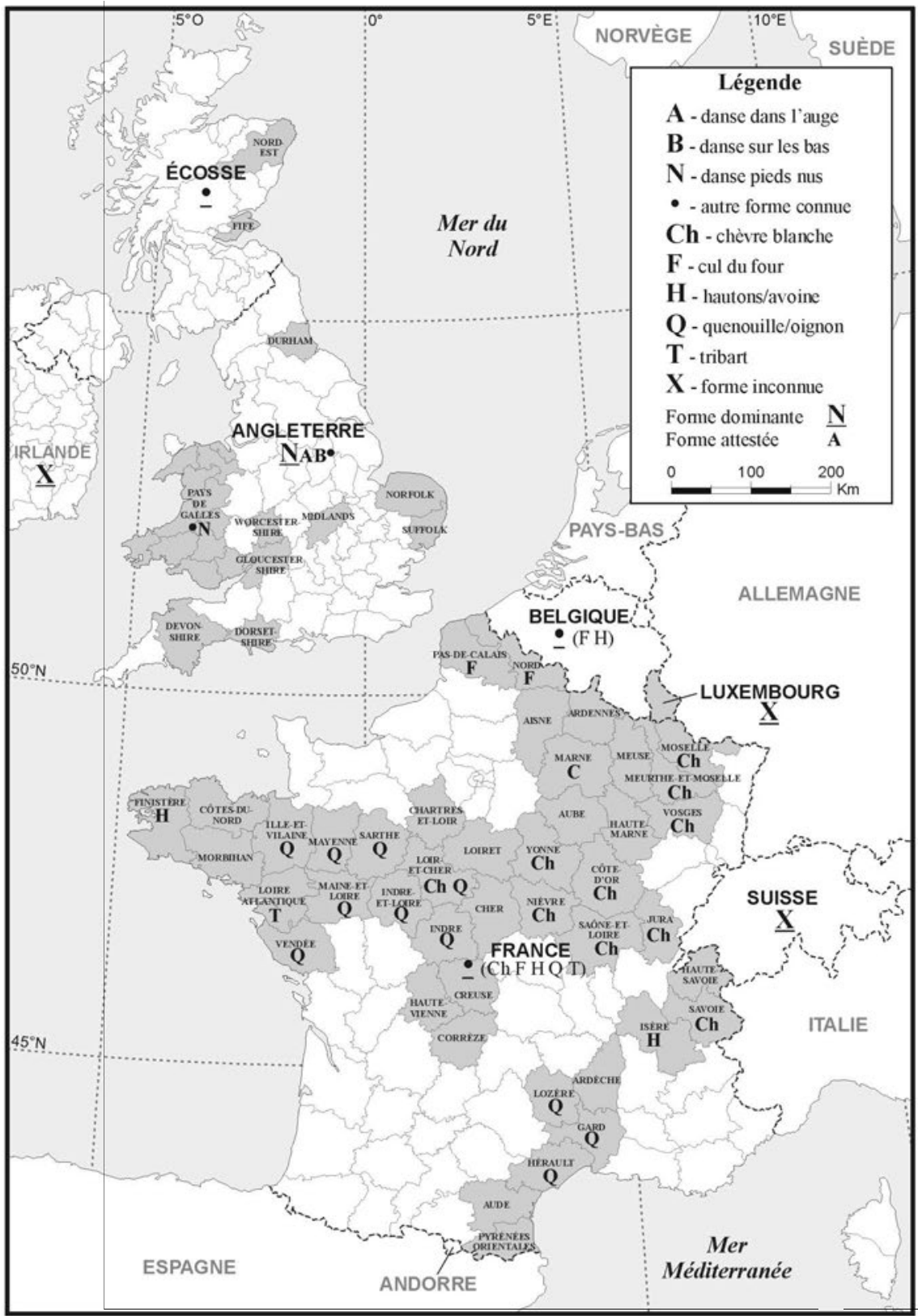


Pologne, Roumanie); on ne la relève pas davantage en Afrique du Nord (Algérie et autres pays du Maghreb, incluant les populations judéo-espagnoles, Égypte, Éthiopie), ni en Afrique noire (Burundi, Cameroun, Côte-d'Ivoire, Sénégal), ni en Asie (Inde). La seule coutume comparable nous a été rapportée du Proche-Orient : à Tripoli, au Liban, les filles célibataires profitent de l'occasion du mariage " pour danser sur la piste nu-pieds, espérant de gagner un mari ${ }^{106} »$; cette pratique n'étant pas destinée à sanctionner les aînées, mais bien à favoriser des rencontres, il ne s'agit donc pas du même rituel.

\section{TABLEAU 3}

Formes de la danse rituelle de l'aîné célibataire selon les époques et les régions d'Europe et d'ailleurs

\begin{tabular}{|c|c|c|c|c|c|}
\hline & 1604 & 1704 & 1804 & 1904 & 2004 \\
\hline \multicolumn{6}{|l|}{ EUROPE } \\
\hline \multicolumn{6}{|l|}{ FRANCOPHONE } \\
\hline France & $\mathrm{X}$ & $\mathrm{X}$ & $\checkmark$ & $\checkmark$ & $\checkmark$ \\
\hline Belgique & $\mathrm{X}$ & $\mathrm{X}$ & $\mathrm{X}$ & $\checkmark$ & $\mathrm{X}$ \\
\hline Suisse & $\mathrm{X}$ & $\mathrm{X}$ & $\mathrm{X}$ & $\mathrm{X}$ & $\mathrm{X}$ \\
\hline \multicolumn{6}{|l|}{ ANGLOPHONE } \\
\hline Angleterre & $\mathrm{N}$ & $\mathrm{N}$ & $\mathrm{N} \boldsymbol{V}_{\mathrm{A}}$ & $\mathrm{AB} \boldsymbol{V}_{\mathrm{N}}$ & $\mathrm{X}$ \\
\hline Écosse & $\mathrm{X}$ & $\mathrm{X}$ & $\checkmark$ & $\mathrm{X}$ & $\mathrm{X}$ \\
\hline Galles & $\mathrm{X}$ & $\mathrm{X}$ & $\checkmark N$ & $\mathrm{X}$ & $\mathrm{X}$ \\
\hline Irlande & $\mathrm{X}$ & $\mathrm{X}$ & $\mathrm{X}$ & $\mathrm{X}$ & $\mathrm{X}$ \\
\hline \multicolumn{6}{|l|}{ AUTRES CONTINENTS } \\
\hline Afrique du Nord & $\mathrm{X}$ & $\mathrm{X}$ & $\mathrm{X}$ & $\mathrm{X}$ & $\mathrm{X}$ \\
\hline Afrique noire & $\mathrm{X}$ & $\mathrm{X}$ & $\mathrm{X}$ & $\mathrm{X}$ & $\mathrm{X}$ \\
\hline Proche-Orient & $\mathrm{X}$ & $\mathrm{X}$ & $\mathrm{X}$ & $\mathrm{X}$ & $\checkmark$ \\
\hline Asie & $\mathrm{X}$ & $\mathrm{X}$ & $\mathrm{X}$ & $\mathrm{X}$ & $\mathrm{X}$ \\
\hline
\end{tabular}

LÉGENDE: A: danse dans l'auge; B: danse sur les bas; $\mathrm{N}$ : danse pieds nus; $\boldsymbol{V}$ : autre forme connue; $\mathrm{X}$ : forme inconnue.

${ }^{106}$ Héba Dabliz, «Les Rites de passage de l'homme de sa naissance à sa mort», rapport d'enquête sur les traditions familiales d'une famille canado-libanaise, Université de Sudbury, janvier 2000, p. 18. 
Mais alors, quel rapport y a-t-il entre ces traditions britanniques et les nôtres? Comment des conduites aussi voisines, dont les noms mêmes sont des expressions qui rappellent de façon troublante les pratiques rituelles canadiennes-françaises, subsisteraient-elles sans un lien direct ou indirect avec les nôtres? Par quel chemin seraientelles venues jusqu'à nous si les Irlandais, les Écossais et les Anglais immigrés au Canada ne les connaissent pas? Comment justifier, si l'on accepte que les Britanniques les aient apportées en Amérique, que, de toutes les populations qui ont été en relation avec eux, seuls les Canadiens français et leurs descendants répandus sur tout le territoire les aient conservées? Pour ce faire, il faudrait prôner quelque aberration: par exemple qu'une amnésie totale ait uniformément frappé tous les descendants d'origine britannique après que leurs ancêtres eussent communiqué cette pratique aux Français d'Amérique; ou encore que ces derniers aient, par une sorte de darwinisme culturel, cette faculté incroyable d'assimiler les traditions des autres peuples dès qu'ils les rencontrent, car, faut-il le répéter, la première mention de cette tradition au Canada remonte au tout début du régime anglais. Voilà de quoi laisser perplexe plus d'un observateur, comme Luc Lacourcière qui s'étonnait de ces transmissions à sens unique dans le domaine du conte populaire. Pour la danse de l'aîné célibataire, force est de trouver une autre voie que la filière britannique. 


\section{TABLEAU 4}

Formes de la sanction rituelle de l'aîné célibataire en Amérique du Nord et en Europe

\begin{tabular}{|c|c|c|c|c|c|c|c|c|c|}
\hline RÉGIONS & 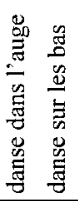 & 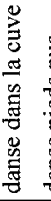 & 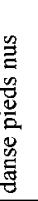 & 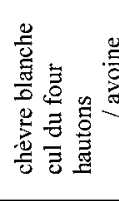 & 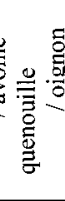 & 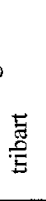 & 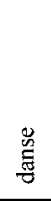 & 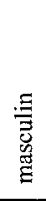 & 䓌 \\
\hline Symboles & A $\mathbf{B}$ & C & & Ch F H & $\mathbf{Q}$ & $\mathbf{T}$ & D & $\sigma^{x}$ & q \\
\hline $\begin{array}{l}\text { AMÉRIQUE DU NORD } \\
\text { Amérique française } \\
\text { Canada } \\
\text { États-Unis }\end{array}$ & $\begin{array}{ll}\mathbf{A} & \mathbf{B} \\
\mathbf{A} & \mathrm{X}\end{array}$ & $\begin{array}{l}\mathbf{C} \\
\mathrm{X}\end{array}$ & $\begin{array}{l}\mathbf{N} \\
\mathrm{X}\end{array}$ & $\begin{array}{lll}X & X & {[H]} \\
X & X & X\end{array}$ & $\begin{array}{l}X \\
X\end{array}$ & $\begin{array}{l}X \\
X\end{array}$ & $\begin{array}{l}\text { D } \\
\text { D }\end{array}$ & $\begin{array}{l}\sigma^{x} \\
\sigma^{x}\end{array}$ & $\begin{array}{l}q \\
q\end{array}$ \\
\hline $\begin{array}{c}\text { Amérique anglaise } \\
\text { Canada } \\
\text { États-Unis }\end{array}$ & $\begin{array}{ll}X & X \\
X & X\end{array}$ & $\begin{array}{l}X \\
X\end{array}$ & $\begin{array}{l}X \\
X\end{array}$ & $\begin{array}{lll}X & X & X \\
X & X & X\end{array}$ & $\begin{array}{l}X \\
X\end{array}$ & $\begin{array}{l}X \\
X\end{array}$ & & & \\
\hline $\begin{array}{l}\text { EUROPE } \\
\text { Europe francophone } \\
\text { France } \\
\text { Belgique } \\
\text { Suisse }\end{array}$ & $\begin{array}{ll}X & X \\
X & X \\
X & X\end{array}$ & $\begin{array}{l}{[\mathrm{C}]} \\
\mathrm{X} \\
\mathrm{X}\end{array}$ & $\begin{array}{l}X \\
X \\
X\end{array}$ & $\begin{array}{lll}\text { Ch } & \mathbf{F} & \mathrm{H} \\
\mathrm{X} & \mathrm{F} & \mathrm{H} \\
\mathrm{X} & \mathrm{X} & \mathrm{X}\end{array}$ & $\begin{array}{l}\mathbf{Q} \\
\mathrm{X} \\
\mathrm{X}\end{array}$ & $\begin{array}{l}T \\
X \\
X\end{array}$ & $\begin{array}{l}{[D]} \\
{[D]}\end{array}$ & $\begin{array}{l}\sigma^{x} \\
\sigma^{x}\end{array}$ & $\begin{array}{l}\text { 우 } \\
\text { ㅇ }\end{array}$ \\
\hline $\begin{array}{c}\text { Europe anglophone } \\
\text { Angleterre } \\
\text { Écosse } \\
\text { Galles } \\
\text { Irlande }\end{array}$ & $\begin{array}{ll}\mathbf{A} & \mathbf{B} \\
\mathrm{X} & \mathrm{X} \\
\mathrm{X} & \mathrm{X} \\
\mathrm{X} & \mathrm{X}\end{array}$ & $\begin{array}{l}\mathrm{C} \\
\mathrm{X} \\
\mathrm{X} \\
\mathrm{X}\end{array}$ & $\begin{array}{l}\mathbf{N} \\
X \\
\mathbf{N} \\
\mathrm{X}\end{array}$ & $\begin{array}{lll}X & X & X \\
X & X & X \\
X & X & X \\
X & X & X\end{array}$ & $\begin{array}{l}X \\
X \\
X \\
X\end{array}$ & $\begin{array}{l}X \\
X \\
X \\
X\end{array}$ & D & $\left|\sigma^{x}\right|$ & 우 \\
\hline
\end{tabular}

Les symboles entre crochets indiquent que cette forme de la tradition n'est pas ordinaire.

\section{B. Le baz-valan et la filière bretonne}

Dans une communication présentée à Sudbury en $1991^{107}$, l'ethnologue brestois Donatien Laurent proposait une piste de recherche porteuse d'éventuelles découvertes. Constatant que les traditions bretonnes et francoontariennes avaient puisé "pour une large part [leurs] racines dans un terreau commun: l'Ouest de la France,

${ }^{107}$ Donatien Laurent, «La Place du conte populaire français d'Amérique dans la tradition orale de la francophonie ", dans L'EEuvre de Germain Lemieux, s.j. Bilan de l'ethnologie en Ontario français. Actes du colloque tenu à l'Université de Sudbury les 31 octobre, $1^{\text {er }}$ et 2 novembre 1991, sous la direction de Jean-Pierre Pichette, Sudbury, Prise de parole et Centre franco-ontarien de folklore, coll. «Ancrages », 1993, p. 275-285. 
voire même la Haute-Bretagne», il concluait par cette remarque finale:

Quant au caractère «celtique», que Paul Delarue reconnaissait au conte canadien et dont il voyait - peut-être un peu rapidement - l'explication dans un apport irlandais récent, il est également présent en Basse Bretagne - ne serait-ce qu'à travers la langue - et constitue lui aussi un trait d'union entre nos deux répertoires narratifs traditionnels qu'il conviendrait sans doute de mieux préciser ${ }^{108}$.

La Bretagne aurait-elle joué un rôle dans la transmission de cette sanction populaire? La chose n'est pas impossible. Population appartenant à la celtitude, apparentée par l'histoire, la langue et les traditions aux populations de la GrandeBretagne où des parallèles de cette pratique ont été consignées, elle aurait pu connaître, elle aussi, les rituels dansés attestés dans cette île depuis Shakespeare jusqu'au milieu du $\mathrm{XIX}^{\mathrm{e}}$ siècle, moment qui paraît en marquer le déclin et présager son évanouissement. Les formes britanniques, matrices présumées des variantes canadiennesfrançaises, n'ont jusqu'ici pas laissé de souvenir en Bretagne, pas plus que dans le reste de la France. Certains signes cependant nous font supposer que les Celtes français auraient pu poser dans cette transmission une action décisive.

Nous l'avons vu, la sanction de l'aîné célibataire, le plus souvent réservée à la vieille fille exclusivement, était, dans la péninsule bretonne comme ailleurs en France, une pratique bien ancrée et elle a donné naissance à des expressions toujours vivantes et à des rituels documentés par l'histoire régionale. Parmi les acteurs qui intervenaient dans ces alliances matrimoniales, il en est un dont le rôle présente quelque rapport avec la pratique sous enquête. Il s'agit de l'entremetteur, du marieur ou arrangeur de mariage, appelé baz-valan en breton ${ }^{109}$, et qui devait permettre ${ }^{108}$ Ibid., p. 285.

${ }^{109}$ Selon Van Gennep, op. cit., tome I, vol. I, p. 269-276, ce courtier jouait le même rôle dans toutes les provinces françaises et chez la plupart des peuples. Plusieurs de ses désignations régionales rappellent des expressions de refus 
aux jeunes gens de «trouver chaussure à leur pied ${ }^{110}$ ». Alexandre Bouët, dans son livre intitulé Breiz-Izel ou Vie des Bretons de l'Armorique et publié en 1835 avec l'assistance de l'illustrateur Olivier Perrin (1761-1832), met en scène deux baz-valans, qui rivalisent dans la conclusion d'une union ${ }^{111}$ :

Nous avons déjà parlé en passant de ces Baz-valans ou entremetteurs qui se recrutent dans deux classes rivales, les tailleurs et les mendiants, et contribuent surtout à donner aux mariages bretons cette physionomie originale qui leur est propre. Ce nom de baz-valan, qui vient de baz, bâton, et de valan, genêt, indique l'usage où ils sont de porter, dans leurs missions amoureuses, une branche de cet arbuste qui fut chez nos pères l'emblême [sic] de l'adresse. Les Baz-valans ont des privilèges de plus d'un genre. Lorsqu'ils ont entamé une négociation conjugale, ils sont nés priés à toutes les cérémonies qui s'ensuivent, au qweladen, aux fiançailles du cabaret, et enfin à la noce. Ces invitations de droit font partie de leur salaire; mais en outre, ils reçoivent des

signalées supra: Bourgogne (Croqueson, Croquavoine, Machavoine), Morvan (Croque-avoine); ailleurs, ces noms rappellent l'insigne spécial qu'il porte: Auvergne (Bâton-Blanc), Bretagne (Bazvalan, Chausse-naire), Normandie (Chausse-noire).

${ }^{110}$ Selon Paul Fortier-Beaulieu, cette expression renverrait plutôt en France au garçon d'honneur, appelé "grand chaussère" dans le Haut-Forez, qui avait " pour mission de mettre à la mariée ses souliers » avant le départ du cortège; ainsi, on disait "de la mariée qu'elle "a trouvé chaussure à son pied" ». Voir Un mariage dans le Haut-Forez en 1873. Récit d'un ménétrier de noces. Publié par Paul Fortier-Beaulieu avec 28 dessins originaux en couleurs de Danielle Murgue, [Paris, Éditions Blondel La Rougery], «Contes des provinces de France" , [1938], p. 45, et du même auteur, Mariages et noces campagnardes, op. cit., "Chaussères", p. 216.

${ }^{111}$ Breiz Izel ou Vie des Bretons dans l'Armorique. Cent-vingt [sic] dessins d'Olivier Perrin avec un texte explicatif par Alexandre Bouët (1835) et une notice sur Olivier Perrin par Alexandre Duval de l'Académie française (1835). Nouvelle édition avec une préface et des notes par Frédéric Le Guyader, conservateur de la Bibliothèque de Quimper, Quimper, J. Salaun, éditeur; Paris, Librairie ancienne E. Champion; Brest, D. Derrien, libraire-éditeur; Rennes, Imprimerie H. Vatar, 1918, XXIV-487 pages. Pour une édition plus récente, voir: Breiz-Izel ou Vie des Bretons de l'Armorique. Texte d'Alexandre Bouët. Dessins d'Olivier Perrin. Présentés et commentés par Mr le Médecin général Ch[arles] Laurent, Mayenne, Joseph Floch, imprimeur-éditeur; Quimper, Société archéologique du Finistère, 1977, XL-278 pages. 
cadeaux en habits ou en argent ${ }^{112}$.

Il précise que ces tâches étaient le lot de miséreux et de gueux qui se situaient tout au bas de l'échelle sociale: les mendiants, qui trouvaient là un moyen d'améliorer leur sort durant quelque temps, et les tailleurs, «des malheureux disgraciés par la nature, des bossus, des borgnes, des boiteux, tout ce qu'il y avait de contrefait et d'incomplet dans la population mâle des campagnes ${ }^{113} »$, qui pouvaient en tirer quelque prestige et faire un moment oublier leur « infériorité physique et leurs occupations féminines». Dans les deux cas, c'étaient des métiers qui mettaient leurs titulaires, ordinairement célibataires, en relation avec de nombreuses personnes et leur permettaient d'intervenir en faveur de leur candidat sans trop éveiller de soupçon; à l'affût de la nouvelle, ils passaient pour «les gazetiers de l'Armorique ${ }^{114} »$. L'auteur prétend que son tailleur était "Baz-valan si adroit, en effet, qu'il eût été capable de marier un turc et une juive, un fakir et un lapon !115

Mais, le succès n'étant pas toujours assuré, la tradition avait prévu des façons de signifier à l'ambassadeur que sa démarche était approuvée ou refusée.

Dans quelques cantons, lorsqu'un Baz-valan vient faire une demande en mariage, on met la poêle sur le feu, et, suivant que

\footnotetext{
${ }^{112}$ Breiz-Izel ou Vie des Bretons de l'Armorique, op. cit., chapitre 87 «Ar c'hemener-bazvalan. - Splihou sant Kirio. Le tailleur en ambassade. Les épingles de saint Kirio», p. 179. Ce mode de rétribution aurait été assez répandu, comme en témoigne Eugène Monseur, "Coutumes. I. — Les Noces", dans Bulletin du folklore wallon, Liège et Bruxelles, vol. III, tome $2,1^{\text {er }}$ fascicule, janvier-mars 1893, p. 18: «1. - Le faiseur de mariages - Dans la partie allemande du Luxembourg belge, un homme, appelé le heiligman, fait profession d'arranger les mariages. Lorsque ses négociations aboutissent, il touche des pour-cent sur la dot et reçoit un chapeau de haute forme et une paire de bottes. Tant qu'elles durent, il vit aus [sic] crochets des deus [sic] familles intéressées, buvant, mangeant et logeant, tantôt chez l'une, tantôt chez l'autre. (La Gazette de Bruxelles du 7 déc. 1890).»

${ }^{113}$ Ibid., p. 180.

${ }^{114}$ Ibid., p. 183.

${ }^{115}$ Ibid., p. 180.
} 
cette demande agrée ou n'est pas accueillie, on pose la poêle comme à l'ordinaire et on fait une omelette qui se mange en famille, ou bien on met ladite poêle le dos en l'air. Cette manière originale de répondre épargne d'un côté l'embarras et de l'autre la mortification d'un refus ${ }^{116}$.

L'ouvrage donne encore d'autres signes d'acceptation ou de refus: d'une part, «ne pas boire avec un Baz-valan, c'est le congédier de la manière la plus formelle ${ }^{117} »$; d'autre part, « remettre au vieux mendiant un tronçon de pain [est un] premier gage de sa satisfaction ${ }^{118} »$ comme les gestes du père qui puise du tabac dans la blague que lui tend le baz-valan et qui l'emmène boire au cellier sont aussi des signes que son ambassade est agréée ${ }^{119}$. Après la signature des accords matrimoniaux au cabaret, l'entremetteur pourra réclamer «l'énorme tranche de lard que lui avait promise la mère de Corentin [le prétendant], le boisseau de seigle qu'il espérait du père, et le broc de vin dont ne manquerait pas de le gratifier Corentin lui-même qui connaissait ses goûts ${ }^{120} »$. Mais là ne s'arrêtait pas la liste des privilèges des baz-valans.

Autrefois les nouveaux mariés leur devaient au moins une paire de bas à coins jaunes; cet usage a disparu ou à peu près, comme celui où ils étaient de chausser, pour aller faire la demande en mariage, un sabot d'un pied et un soulier de l'autre, ou bien

\footnotetext{
${ }^{116}$ Ibid., chapitre 88 «Ar paour-bazvalan. - Le mendiant en ambassade», p. 181.

${ }^{117}$ Ibid., p. 182. Van Gennep, op. cit., tome I, vol. I, p. 272, note aussi des refus symboliques contemporains, soit par des ustensiles renversés: balai (Pays messin et Auvergne); marmite (Basse-Normandie); ou des gestes: mettre dans la poche une poignée d'avoine (Isère, Basses-Alpes), de la braise ou des tisons; offrir une omelette marque le refus, de la viande exprime l'acceptation. «Plus rare est l'envoi au galant dont on ne veut pas d'un animal, coutume presque partout disparue, mais qui subsiste par allusion dans quelques dictons. En Bretagne on lui envoyait une chèvre, en Mâconnais un chat, de même en Lorraine. Peut-être faut-il rapprocher ce détail de l'envoi d'une chèvre ou d'un autre animal à l'aînée lorsque la cadette se marie avant elle» (p. 275). ${ }^{118}$ Loc. cit.

${ }^{119}$ Ibid., chapitre 90 "Ann divezou kenta. - Ar re-all e spi. Les premiers accords. - L'embuscade», p. 186.

${ }^{120}$ Ibid., chapitre 91 «Distro ar c'hlasker-bara. - Ann divezou en hostaliri. Le retour du mendiant. - Les accords au cabaret», p. 187.
} 
encore de porter, en pareil cas, un bas rouge et un bas bleu. Ces attributs grotesques appartenaient aux tailleurs qui se faisaient Baz-valans, et sont conformes à l'esprit de servilité bouffonne qui en a fait une classe à part ${ }^{121}$.

\section{LA DANSE SUR LES BAS}

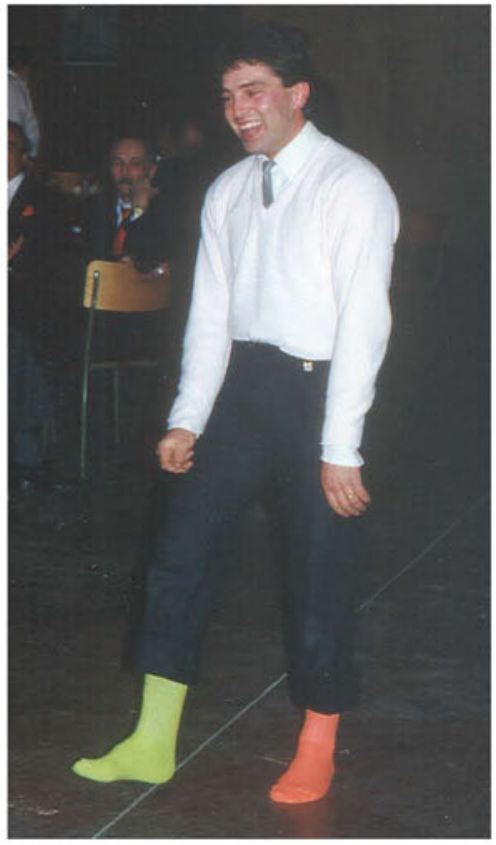

Pното 1: L'aîné (Richard Couture) dansant sur ses bas (un vert et un rouge), Ville des Laurentides (Québec), 2 février 1986.

Source : Danielle Couture-Haché (Dieppe, Nouveau-Brunswick).

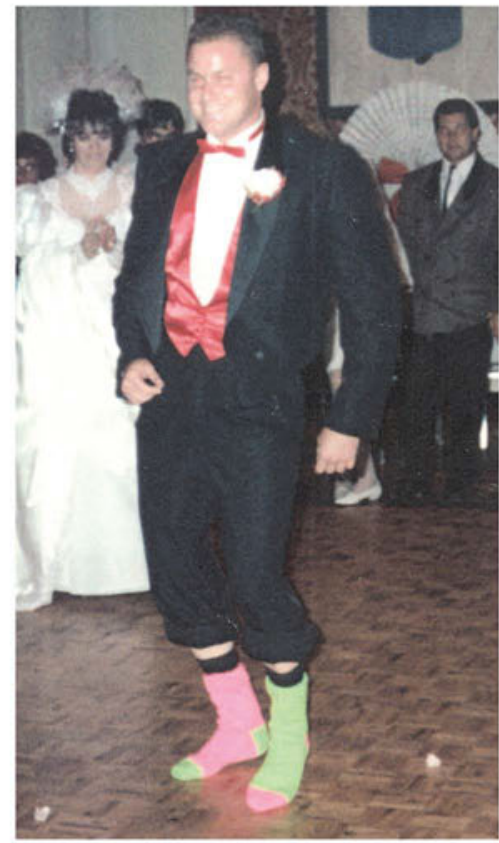

Pното 2: L'aîné (Ronald Hogue) dansant sur ses bas (un rose aux extrémités vertes et un vert aux extrémités roses), Hanmer (Ontario), août 1991. Source: Ronald Hogue (Hanmer, Ontario).

Jean-Michel Guilcher a même relevé, au cours de ses enquêtes en Basse-Bretagne, une variété particulière de récompense, conservée localement, et qui prenait la forme d'une danse:

${ }^{121}$ Ibid., chapitre 87 "Ar c'hemener-bazvalan. - Splihou sant Kirio. Le tailleur en ambassade. Les épingles de saint Kirio», p. 179. 
En pays du Faouët et de Guémené sur Scorff l'entremetteur de mariage (c'est-à-dire l'homme qui a servi d'intermédiaire entre les deux familles) a droit à une ou deux danses avec la mariée. Le premier jour il mène une gavotte avec elle. C'est souvent la première après la danse d'honneur. Le second jour il se fait le boute-en-train et amuseur. Affublé d'une peau de bête et d'accessoires divers, il est censé représenter une chèvre, un bouc ou un cheval, suivant le sobriquet local qu'on lui donne. If Il est littéralement couvert de gâteaux, sucreries, et friandises de toutes sortes, suspendues à des ficelles qu'il porte en sautoir. Il les défend contre les gourmands jusqu'au grand assaut final que ceux-ci lui donnent pendant ou après une nouvelle danse avec la mariée. Ce thème de jeu, prétexte à improvisations comiques de toutes sortes, paraît ne remonter qu'à la fin du siècle dernier ${ }^{122}$.

Ce personnage de l'entremetteur ou baz-valan, ordinairement célibataire, qui portait dans l'exercice de sa charge des chaussettes de couleurs différentes - un bas rouge et un bas bleu - et pouvait réclamer de la mariée, une fois l'accord intervenu, une ou plusieurs danses au cours du bal des noces ${ }^{123}$, aurait-il quelque chose à voir avec la nature et le moment de la sanction, avec la victime et la couleur des bas qu'elle doit porter dans la tradition franco-ontarienne? Cette hypothèse ne pourra guère se vérifier tant qu'on ne possédera pas la preuve de liens plus étroits entre la tradition nord-américaine et la tradition bretonne ou française. Les bas désassortis du valet mis en scène par Shakespeare - bas de fils jarreté d'un cordon bleu apparié à une chausse de laine jarretée de rouge - ont beau rappeler la tenue carnavalesque du baz-valan breton - chaussé d'un sabot

${ }^{122}$ Jean-Michel Guilcher, op. cit., p. 37.

${ }^{123}$ Van Gennep, op. cit., tome I, vol. 1, 1943, p. 269-276. Voir aussi Martine Segalen avec la collaboration de Josselyne Chamarat, Amours et mariages de l'ancienne France, [Paris], Bibliothèque Berger-Levault, "Arts et traditions populaires", [1981], p. 85 et 87 : dans la légende accompagnant le dessin du Breiz-Izel, elle écrit: "Appelé parfois du nom de «Baz-valan », d'après le bâton de genêt qu'il porte tel un embl[è]me, l'entremetteur se distingue aussi par des bas de couleurs différentes, l'un rouge, l'autre violet [sic]». Bernadette de Paimpont, Bretagne éternelle, [Arts et traditions]. [Lausanne], Edita S.A., [1996], p. 51: «le Baz-Valan s'arme de la branche de genêt, qui lui donne ce nom, et, chaussé d'un bas rouge et d'un bas bleu [...]». 
d'un pied et d'un soulier de l'autre, ou d'un bas rouge et d'un bas bleu - et les bas loufoques de l'aîné francoontarien - parfois l'un rouge ou rose, l'autre brun ou vert, souvent à bandes multicolores qui se répètent inversement d'un bas à l'autre -, la démonstration reste incomplète. La lettre de Jean-Michel Guilcher, homme de terrain et spécialiste de la danse, nous donne tout lieu d'espérer pouvoir compléter un jour cette preuve. Répondant à notre demande de renseignements, il écrit:

aucune de nos enquêtes de terrain, conduites dans quantité de régions de France, ne nous a fait connaître, encore présent dans la mémoire de nos informateurs, le genre d'usage auquel vous faites allusion. Il a existé pourtant des pratiques de cette sorte; je me rappelle en avoir vu évoquées, voilà longtemps, dans des récits de témoins anciens. Je ne saurais de mémoire préciser où ${ }^{124}$.

Il confirmait en outre les différences remarquées: la victime est constamment la fille aînée et la danse n'est pas un trait obligatoire, tandis que la chèvre et le four le sont. La distance est énorme entre nos traditions et la filiation pour l'instant bien énigmatique. Pourra-t-on jamais trouver mention en Bretagne ou dans l'ouest de la France de la présence de cette forme du rituel quand Bouët note au Breiz-Izel, en 1835 , que le don d'une paire de bas à coins jaunes au bazvalan comme le port par celui-ci de bas dépareillés avaient pris le chemin des oubliettes? Des liens ténus subsistent tout de même, entre la Grande-Bretagne et la Bretagne, dans des formes marginales comme ces objets furtivement attachés à la taille et au bras de l'aîné - des jarretières en Écosse, des sachets d'avoine ou hautons en France - , une plaisanterie qu'on jugera consommée si le célibataire les porte ou même danse sans les avoir remarqués. L'expression "porter le fagot d'ajoncs", notée dans le Bas-Léon en 2002 pour désigner la fille aînée supplantée, serait-elle la survivance métaphorique d'un rituel dansé, également ${ }^{124}$ Jean-Michel Guilcher, lettre de Tromao (Esquibien, France), 26 juillet 1999, 3 p. ms. 
réservé à la fille aînée, — «danser sur un fagot d'ajoncs »qui fut attesté dans le centre de l'Angleterre en 1922?

\section{TABLEAU 5}

Comparaison entre la conduite de l'aîné célibataire en Amérique française et celle du baz-valan en Bretagne le jour de la noce

\begin{tabular}{|c|c|c|c|}
\hline ÉLÉMENTS & Communs & \multicolumn{2}{|c|}{ DIVERGENTS } \\
\hline \multicolumn{2}{|l|}{ ACTEURS } & Aîné & Baz-valan \\
\hline Statut & célibataire & aîné non marié & $\begin{array}{l}\text { entremetteur: } \\
\text { mendiant ou tailleur }\end{array}$ \\
\hline Moment & jour du mariage & \multicolumn{2}{|l|}{ au bal des noces } \\
\hline \multirow[t]{2}{*}{ Nature } & danse & $\begin{array}{l}\text { en solitaire } \\
\text { [en couple : encouragement } \\
\text { passager de la mariée ou } \\
\text { d'une autre invitée] }\end{array}$ & $\begin{array}{l}\text { en couple } \\
\text { avec la mariée } \\
\text { [1 ou } 2 \text { danses] }\end{array}$ \\
\hline & activité & obligatoire & accessoire \\
\hline \multirow[t]{3}{*}{ Costume } & bas multicolores & \multirow{2}{*}{$\begin{array}{l}\text { un brun (vert) et un rouge } \\
\text { (rose) / à bandes colorées } \\
\text { répétées à l'inverse d'un bas } \\
\text { à l'autre / à ornementation } \\
\text { loufoque }\end{array}$} & \multirow{2}{*}{$\begin{array}{l}\text { un bleu et un rouge } \\
\text { [portés dans son rôle } \\
\text { d'entremetteur] } \\
\text { bas à coins jaunes en } \\
\text { présent }\end{array}$} \\
\hline & allure grotesque & & \\
\hline & $\begin{array}{l}\text { chaussures } \\
\text { inconfortables }\end{array}$ & $\begin{array}{l}\text { [inconfort de la danse dans } \\
\text { l'auge : un pied dedans, } \\
\text { l'autre dehors] }\end{array}$ & $\begin{array}{l}\text { un pied dans un sabot, } \\
\text { l'autre dans un soulier }\end{array}$ \\
\hline \multirow[t]{2}{*}{ Objet } & divertissement & $\begin{array}{l}\text { expiation obligée d'une } \\
\text { infraction }\end{array}$ & $\begin{array}{l}\text { récompense : } \\
\text { obtention d'un } \\
\text { privilège }\end{array}$ \\
\hline & résultat public & échec (humiliation) & succès (promotion) \\
\hline \multirow[t]{2}{*}{ Tradition } & actualité & $\begin{array}{l}\text { contemporaine et en pleine } \\
\text { vitalité }\end{array}$ & $\begin{array}{l}\text { disparue depuis près } \\
\text { de deux siècles }\end{array}$ \\
\hline & déroulement & $\begin{array}{l}\text { bien documenté et très } \\
\text { élaboré }\end{array}$ & peu documenté \\
\hline
\end{tabular}

\section{ÉPILOGUE}

À la lumière de ce qui précède, il est certain que les deux formes principales de cette sanction, danser sur ses bas et danser dans l'auge, ne sont pas nées au Canada. En ce qui concerne l'ordre du mariage dans les familles, le respect du droit de primogéniture est universel et le jour de la noce, 
l'une des occasions où il se manifeste le plus fortement. L'existence de rituels où l'on fustige symboliquement l'aîné non marié est confirmée en France et dans les états limitrophes de la francophonie de l'Europe occidentale, mais aussi dans les îles Britanniques, régions où nous avons concentré nos investigations. Or, en Amérique du Nord, ces traditions ne sont présentes que dans la francophonie et, curieusement, les formes européennes les plus approchantes ont été relevées en Grande-Bretagne. La recherche du maillon manquant, celui qui expliquerait le passage de ces formes singulières vraisemblablement celtes vers la francophonie nord-américaine, ne saurait reposer exclusivement sur la présence de ressortissants d'origine britannique au Canada - Anglais, Écossais, Irlandais, Gallois chez qui ces traditions n'ont reçu jusqu'à présent aucun écho, mais elle devrait plutôt scruter en priorité les populations celtes continentales, les Bretons en particulier, dont les affinités avec la culture celte en même temps qu'avec la culture française pourraient bien élucider cet apparent mystère d'une tradition qui n'a pu migrer ici qu'avec les colons français, tout simplement.

Dans les circonstances, il serait opportun d'examiner la nature et la qualité de l'apport français au patrimoine canadien afin de connaître l'originalité et la spécificité de chacune de ces deux traditions. Ce faisant, il conviendrait de mesurer plus particulièrement l'étendue de la contribution des Celtes en cette matière et de statuer sur sa réalité ou sur sa virtualité. Ainsi, on pourrait analyser les raisons du maintien, de l'adaptation, du renouvellement ou, au contraire, de l'oubli ou de l'absence de certains types français en Bretagne et au Canada; ces travaux mettraient en valeur l'apport de la Bretagne et de l'ouest de la France à la constitution de ce répertoire d'outre-mer en reconsidérant, par exemple, le lieu, en France déjà ou au Canada, de l'influence celtique reconnue par Delarue. La double appartenance de la Bretagne à la culture des pays 
celtes et à la culture de la francophonie expliquerait-elle le cheminement d'un certain nombre des traditions canadiennes - surtout celles qui n'ont pas laissé de trace en France, mais qui sont attestées chez les Celtes d'Irlande, d'Écosse ou d'ailleurs? En choisissant bien un certain nombre de traditions (orales, coutumières, matérielles) bretonnes et canadiennes selon des critères pertinents (leur haute fréquence, leur présence commune ou, au contraire, leur absence dans l'un ou l'autre des terroirs, y compris ceux des autres pays celtiques, leur dépendance ou leur indépendance par rapport à des sources littéraires, par exemple), il serait possible d'obtenir des résultats probants sur ces questions fascinantes. C'est donc à l'investigation de cette dynamique, aux enjeux identitaires fort apparents, mais dont le cheminement en ces divers terrains reste à confirmer, qu'invite cette étude.

\section{REMERCIEMENTS}

En plus de nos collègues de la Société Charlevoix, qui ont tous lu et commenté judicieusement cette étude, nous voulons remercier sincèrement chacune des personnes dont les noms suivent, car leurs références nombreuses et leurs observations l'ont considérablement enrichie: Nancy-Gaëlle Barras (Chermignon), Adrienne Boulvin (Bruxelles), Giovanni Battista Bronzini† (Bari), Francine Brunel-Reeves (Montréal), Judith R. Cohen (Toronto), Estelle Corriveau (Hanmer), Danielle Couture-Haché (Dieppe), T.G.O. Crualaoich (Cork), Héba Dabliz (Tripoli, Sudbury), Luisa Del Giudice (Los Angeles), Fritz-Gérald Destiné (Sudbury), J.J. Dias Marques (Portugal), Martine Duquesne (Paris), Barbara Fernandez Taviel de Andrade (Ciudad Real), Frances J. Fisher (Édimbourg), Annie Gagné (Cornwall), Paulette Galand-Pernet (Paris), Daniel Giraudon (Brest), Pauline Greenhill (Winnipeg), Jean-Michel Guilcher (Esquibien), Carol Harvey (Winnipeg), Ronald Hogue (Hanmer), Camille Lacoste-Dujardin (Paris), Sophie Laflamme (Hearst), Donatien Laurent (Brest), Maria Santa Montez (Portugal), Éding Mvilongo (Sudbury), W.F.H. Nicolaisen (Aberdeen), Roger Pinon (Liège), Fañch Postic (Mellac), Jean-François Simon (Brest), Marie-Rose Simoni-Aurembou (Paris), Michael Taft (Washington), André Thill (Paris), Stefaan Top (Louvain), Jacinthe Trudeau (Saint-Charles), Diane Tye (Saint-Jean, Terre-Neuve), Rionach ui Ogain (Dublin), Évelyne Voldeng† (Ottawa), Peter Ward (Vancouver), J.D.A. Widdowson (Londres), Fionnuala Williams (Belfast). 